\title{
The High Energy Telescope for STEREO
}

\author{
T.T. von Rosenvinge $\cdot$ D.V. Reames $\cdot$ R. Baker $\cdot$ J. Hawk $\cdot$ J.T. Nolan $\cdot$ L. Ryan • \\ S. Shuman • K.A. Wortman · R.A. Mewaldt • A.C. Cummings • W.R. Cook • \\ A.W. Labrador · R.A. Leske • M.E. Wiedenbeck
}

Received: 1 May 2007 / Accepted: 18 December 2007 / Published online: 14 February 2008

(C) Springer Science+Business Media B.V. 2008

\begin{abstract}
The IMPACT investigation for the STEREO Mission includes a complement of Solar Energetic Particle instruments on each of the two STEREO spacecraft. Of these instruments, the High Energy Telescopes (HETs) provide the highest energy measurements. This paper describes the HETs in detail, including the scientific objectives, the sensors, the overall mechanical and electrical design, and the on-board software. The HETs are designed to measure the abundances and energy spectra of electrons, protons, $\mathrm{He}$, and heavier nuclei up to Fe in interplanetary space. For protons and He that stop in the HET, the kinetic energy range corresponds to $\sim 13$ to $40 \mathrm{MeV} / \mathrm{n}$. Protons that do not stop in the telescope (referred to as penetrating protons) are measured up to $\sim 100 \mathrm{MeV} / \mathrm{n}$, as are penetrating He. For stopping $\mathrm{He}$, the individual isotopes ${ }^{3} \mathrm{He}$ and ${ }^{4} \mathrm{He}$ can be distinguished. Stopping electrons are measured in the energy range $\sim 0.7-6 \mathrm{MeV}$.
\end{abstract}

Keywords Space instrumentation · STEREO mission · Energetic particles · Coronal mass ejections $\cdot$ Particle acceleration

PACS 96.50.Pw $\cdot 96.50 . \mathrm{Vg} \cdot 96.60 . \mathrm{ph}$

\section{Abbreviations}

2-D Two dimensional

ACE Advanced Composition Explorer

ACRs Anomalous Cosmic Rays

ADC Analog to Digital Converter

T.T. von Rosenvinge $(\bowtie) \cdot$ D.V. Reames $\cdot$ R. Baker $\cdot$ J. Hawk $\cdot$ J.T. Nolan $\cdot$ L. Ryan · S. Shuman · K.A. Wortman

NASA/Goddard Space Flight Center, Greenbelt, MD 20771, USA

e-mail: Tycho.T.vonRosenvinge@nasa.gov

R.A. Mewaldt · A.C. Cummings · W.R. Cook · A.W. Labrador · R.A. Leske

California Institute of Technology, Pasadena, CA 91125, USA

M.E. Wiedenbeck

Jet Propulsion Laboratory, California Institute of Technology, Pasadena, CA 91109, USA 
ApID Application Identifier; used to identify specific CCSDS packets

APL The Applied Physics Laboratory of Johns Hopkins University

Calstim Calibration stimulus event

Caltech California Institute of Technology

CCSDS Consultative Committee for Space Data Systems

CME Coronal Mass Ejection

CPU Central Processor Unit

CPU24 GSFC-specific version of MISC

DAC Digital-to-Analog Converter

EEPROM Electronically Erasable Programmable Read-Only Memory

EPAM Electron, Proton, and Alpha Monitor (on the ACE spacecraft)

ETU Engineering Test Unit (prototype)

FIFO First In First Out (event queues)

FM1 Flight Model 1 (Ahead spacecraft)

FM2 Flight Model 2 (Behind spacecraft)

FPGA Field Programmable Gate Array

GCRs Galactic Cosmic Rays

GEANT Geometry ANd Tracking toolkit for the simulation of particle instruments

GOES Geostationary Operational Environmental Satellite (NOAA)

GOR gated OR of binary signals

GSFC Goddard Space Flight Center

HET High Energy Telescope

HVPS High Voltage Power Supply

I/F Interface

I/O Input/Output

ICD Interface Control Document

IDPU Instrument Data Processing Unit

IMP-8 Interplanetary Monitoring Platform number 8

IMPACT In situ Measurements of Particles and CME Transients (title of the STEREO investigation to which HET belongs)

JPL Jet Propulsion Laboratory

LET Low Energy Telescope

MISC Minimal Instruction Set Computer

MOC Mission Operations Center

MRD Mission Requirements Document

MSU Michigan State University

$\mathrm{MSb} \quad$ Most Significant bit

NASA National Aeronautics and Space Administration

NOAA National Oceanic and Atmospheric Administration

NSCL National Superconducting Cyclotron Laboratory

OSO Orbiting Solar Observatory

PDFE Particle Detector Front End

PH Pulse Height

PHA Pulse Height Analyzer

PHASIC Pulse Height Analysis System Integrated Circuit

POC Payload Operations Center

PREOUT analog output of a PHASIC preamplifier selected by command

RNDN Signal that is high during the run-down of a Wilkinson pulse-height converter SAMPEX Solar, Anomalous, and Magnetospheric Particle Explorer 


$\begin{array}{ll}\text { SEPs } & \text { Solar Energetic Particles } \\ \text { SEPT } & \text { Solar Electron Proton Telescope } \\ \text { SEU } & \text { Single Event Upset (flipping of a bit by a highly ionizing particle) } \\ \text { SIT } & \text { Suprathermal Ion Telescope } \\ \text { SoHO } & \text { Solar and Heliospheric Observatory } \\ \text { SRAM } & \text { Static Random Access Memory } \\ \text { SSC } & \text { STEREO Science Center } \\ \text { SSD } & \text { Solid-State Detector } \\ \text { STEREO } & \text { Solar-TErrestrial RElations Observatory } \\ \text { stim } & \text { electronically stimulated event } \\ \text { TOF } & \text { Time of Flight } \\ \text { UART } & \text { Universal Asynchronous Receiver/Transmitter } \\ \text { UCB } & \text { University of California at Berkeley } \\ \text { UK } & \text { United Kingdom (England) } \\ \text { VDA } & \text { Vacuum-Deposited Aluminum } \\ \text { VLSI } & \text { Very Large Scale Integration }\end{array}$

\section{Introduction}

\subsection{STEREO/IMPACT/SEP/HET}

NASA's Solar Terrestrial Relations Observatory (STEREO) comprises two nearly identical observatories in orbit about the Sun (one gradually moving ahead of Earth, called the Ahead spacecraft, and the second gradually moving behind, called the Behind spacecraft). STEREO will provide the first 3-D images of the solar corona and of coronal mass ejections (CMEs). These stereo images will be supplemented by multi-point in situ measurements of solar wind and CME plasma and of the energetic particles accelerated in association with CME-driven shocks and solar flares. The High Energy Telescope (HET) is one of four instruments in the Solar Energetic Particle (SEP) subsystem that is part of the IMPACT (InSitu Measurements of Particles and CME Transients) investigation on STEREO. IMPACT is providing measurements of solar wind and suprathermal electrons, interplanetary magnetic fields, and solar energetic particles (Luhmann et al. 2007, 2005). The SEP suite is composed of the Solar Electron Proton Telescope (SEPT; Müller-Mellin et al. 2007), the Suprathermal Ion Telescope (SIT; Mason et al. 2007), the Low-Energy Telescope (LET; Mewaldt et al. 2007b), and the HET.

The STEREO Mission Requirements Document (MRD) includes the following Science Objective that is specifically directed toward energetic particle studies:

Discover the mechanisms and sites of energetic particle acceleration in the low corona and the interplanetary medium.

The four SEP instruments address this objective by providing complementary measurements of the composition and energy spectra of energetic nuclei from $\mathrm{H}$ to $\mathrm{Fe}(1 \leq Z \leq 26)$ that span the energy range from $\sim 0.03$ to $>100 \mathrm{MeV} / \mathrm{n}$, as well as measurements of electrons from 0.03 to $6 \mathrm{MeV}$ (see Fig. 1). Note that the HET is responsible for measuring the highest energy particles observed by these four sensors. STEREO will also make an important contribution to space weather forecasting efforts by continuously broadcasting Beacon data that include low-resolution coronal and CME images, solar wind and interplanetary magnetic field parameters, and a fairly complete survey of SEP intensities and composition, including protons from HET in the critical energy range from 14 to $>100 \mathrm{MeV}$. 
Fig. 1 Energy coverage by IMPACT/SEP for different species

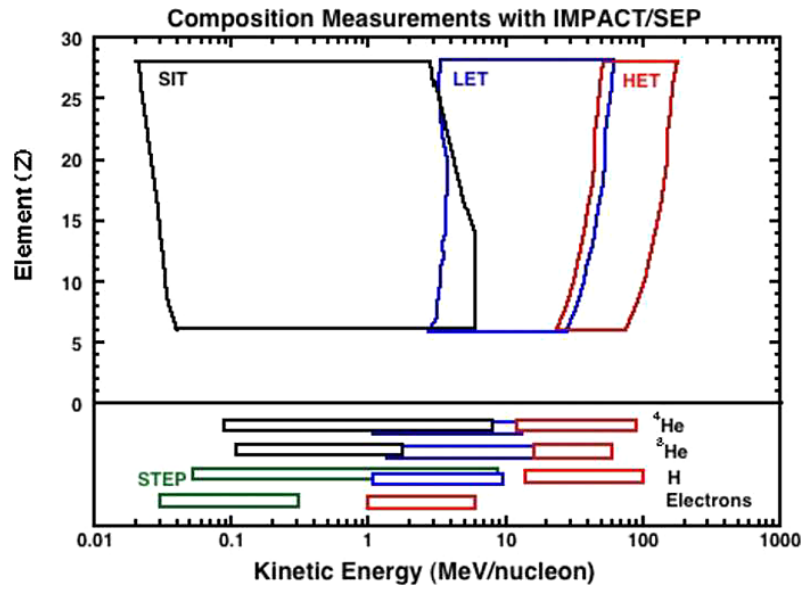

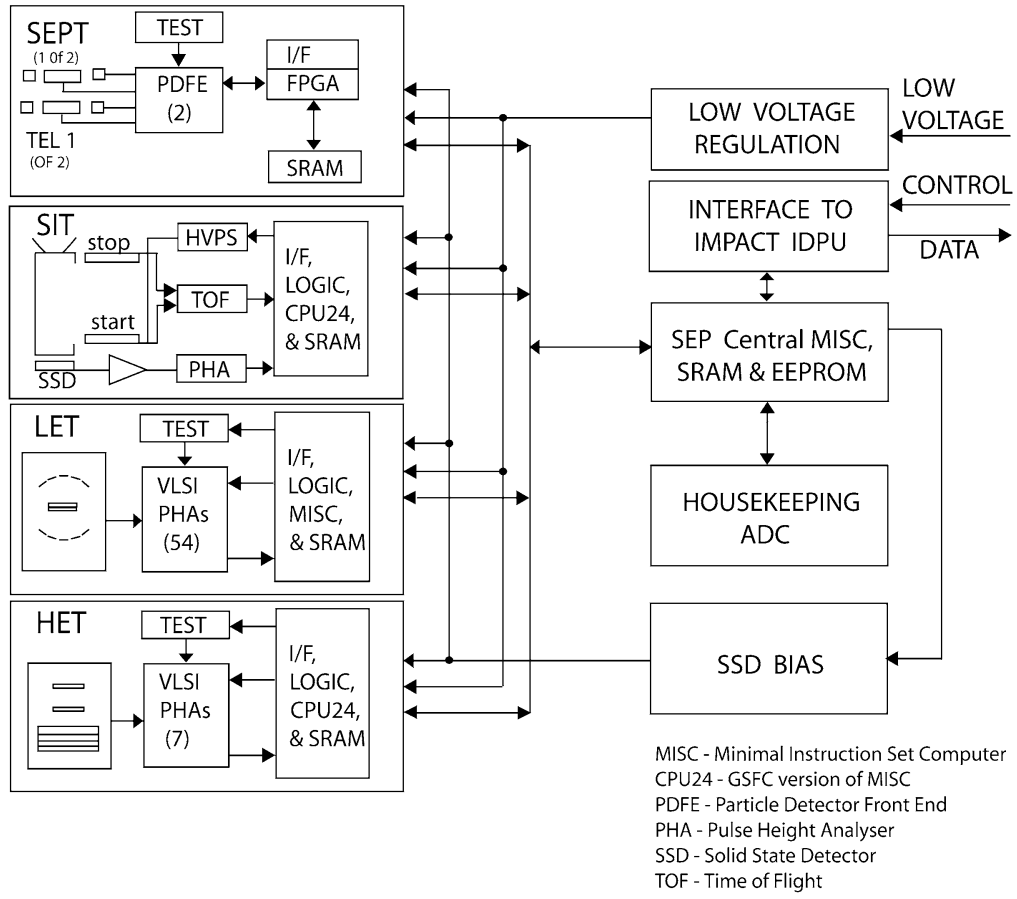

Fig. 2 Block diagram of IMPACT/SEP

A block diagram of the SEP system is shown in Fig. 2. The LET, HET, and SIT sensors each include a dedicated microprocessor for onboard data processing and sensor control. There is an additional control unit called SEP Central (Mewaldt et al. 2007b) that gathers data from the four SEP sensors, controls the SEP bias supply, and manages the interfaces to the sensors and the SEP interface to the Instrument Data Processing Unit (IDPU). The IDPU is described in Luhmann et al. (2007). A photo of HET, LET, and SEP Central is shown in Fig. 3. 
Fig. 3 Photograph of LET (top center), HET (center left) and SEP Central (enclosure at bottom). Overall height is approximately $43.4 \mathrm{~cm}$. The bottom of SEP Central is elevated $3 / 4^{\prime \prime}(1.9 \mathrm{~cm})$ above the spacecraft deck due to thermal isolators

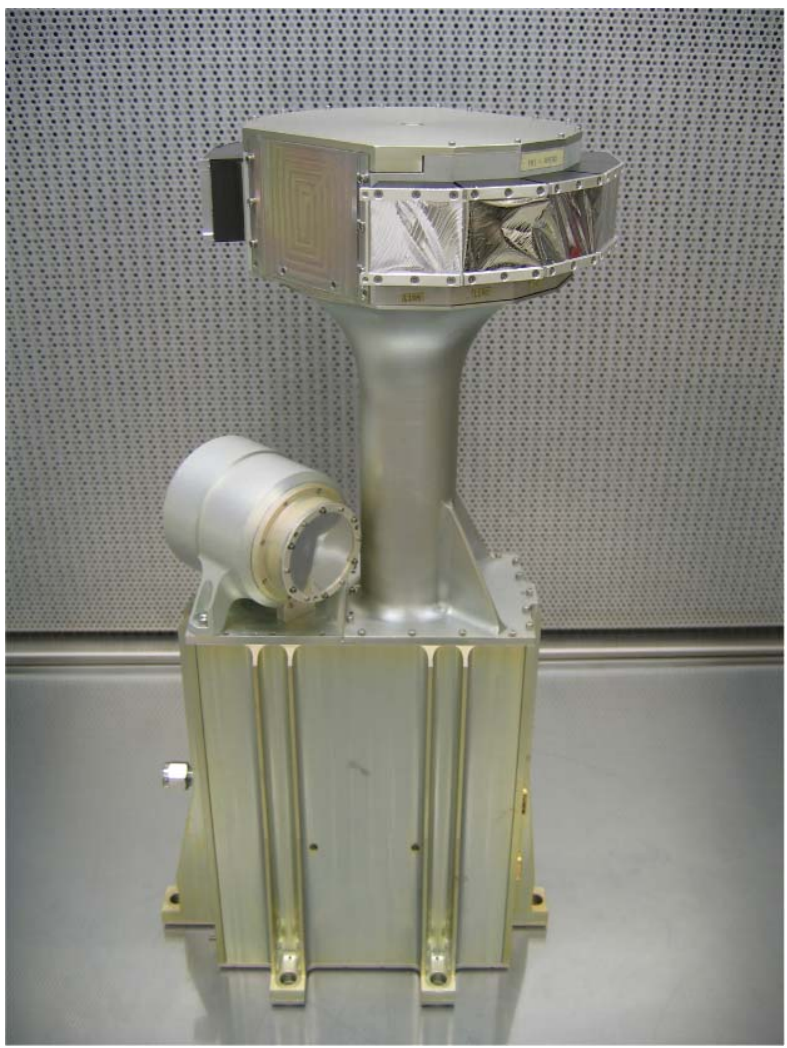

This article describes the HET instrument, along with the science objectives, design requirements, measurement capabilities, and data products. Also described are the onboard software and functions that can be altered by command or with new table uploads. There are two identical HET instruments: Flight Model 1 (FM1) is flying on the STEREO Ahead spacecraft, and FM2 is on the Behind spacecraft. Table 1 summarizes key characteristics of the HET instrument and refers to the sections, figures, and tables where additional details can be obtained. The mounting of HET on each of the two spacecraft is shown in Figs. 4a and $4 \mathrm{~b}$.

The primary measurement goal of HET is to measure the energy spectra and time variations of solar energetic particles, including $\mathrm{H}$ and $\mathrm{He}$ ions with 13 to $100 \mathrm{MeV} / \mathrm{n}$ and electrons with 0.7 to $6 \mathrm{MeV}$. In addition, HET will provide measurements of individual ions ranging from $\mathrm{C}$ to $\mathrm{Fe}$ with $\sim 30$ to $\sim 150 \mathrm{MeV} / \mathrm{n}$ (depending on species; see Fig.1). During solar quiet times HET will also measure the composition and energy spectra of anomalous cosmic ray (ACR) and galactic cosmic ray (GCR) nuclei.

\subsection{Scientific Goals}

The scientific goals of the STEREO mission are centered on better understanding Coronal Mass Ejections (CMEs): what triggers their eruption, what is their role in accelerating energetic particles, how do they affect the transport of particles from the Sun to $1 \mathrm{AU}$, and how do they contribute to magnetospheric storms at Earth? The first CME was observed in 
Table 1 Summary of HET characteristics

\begin{tabular}{|c|c|c|}
\hline Characteristic & Value & Details $^{*}$ \\
\hline Measurement objective & $\begin{array}{l}\text { Composition, energy spectra, and time variations } \\
\text { of solar and interplanetary ions and electrons }\end{array}$ & $\begin{array}{l}\S 1.1 \\
\text { F1-1-1-2 }\end{array}$ \\
\hline Measurement technique & Multiple- $\Delta E$ vs. residual energy & $\S 2$ \\
\hline \multicolumn{3}{|l|}{ Sensor system } \\
\hline Energy-loss measurements & $\begin{array}{l}\text { A single detector stack composed of } 9 \\
\text { ion-implanted Si detectors, each } 1 \mathrm{~mm} \\
\text { in thickness }\end{array}$ & $\begin{array}{l}\S 2.1,2.1 .1,2.1 .2 \\
2.2 ; \mathrm{F} 2.2-\mathrm{F} 2.6\end{array}$ \\
\hline $\begin{array}{l}\text { On-board particle } \\
\text { identification }\end{array}$ & $\begin{array}{l}9 \text { species from } \mathrm{H} \text { to } \mathrm{Fe} \text { with an average } \\
\text { of } 9 \text { energy intervals. Electrons in } 3 \\
\text { energy intervals }\end{array}$ & $\begin{array}{l}\S 3.4 ; \mathrm{A} ; \mathrm{F} 1.1, \mathrm{~F} 2.1 \\
\mathrm{~F} 3.1-3.3, \mathrm{~T} 3.3\end{array}$ \\
\hline Charge interval & Ions with $1 \leq Z \leq 26$; electrons & $\S 2.4 ; \mathrm{F} 1.1, \mathrm{~F} 2.1, \mathrm{~F} 3.2$ \\
\hline Energy interval $(\mathrm{MeV} / \mathrm{n})$ & & $\S 2.4 ; \mathrm{F} 1.1, \mathrm{~F} 2.1$ \\
\hline Electrons & $0.7-6$ & \\
\hline $\mathrm{H}, \mathrm{He}$ & $13-100$ & \\
\hline $\mathrm{O}$ & $28-89$ & \\
\hline $\mathrm{Si}$ & $38-124$ & \\
\hline $\mathrm{Fe}$ & $49-167$ & \\
\hline Field of view & $55^{\circ}$ cone (full angle) & F2.6 \\
\hline Geometry factor & $0.61 \mathrm{~cm}^{2} \mathrm{sr}$ & $\S 2.4$ \\
\hline Event yields (large SEP event) & $>10^{5} \mathrm{H} \& \mathrm{He}$ & $\S 5.3$ \\
\hline Element resolution & $<0.2$ charge units & $\$ 5.3$ \\
\hline Mass resolution & $0.2 \mathrm{amu}$ for ${ }^{3} \mathrm{He}$ and ${ }^{4} \mathrm{He}$ & $\$ 5.3$ \\
\hline Resource allocation & & $\S 4$ \\
\hline Dimensions $(1 \times \mathrm{w} \times \mathrm{h})$ & $\begin{array}{l}\text { Envelope: } 172 \mathrm{~mm} \times 95 \mathrm{~mm} \times 206 \mathrm{~mm} \\
\text { Telescope: } 82 \mathrm{~mm} \text { long } \times 68.5 \mathrm{~mm} \text { diam }\end{array}$ & \\
\hline
\end{tabular}

*Sections (§), figures $(\mathrm{F})$, tables $(\mathrm{T})$ and appendices $(\mathrm{A})$ containing additional details

1971 from OSO-7 (Tousey 1973), and they were subsequently observed by Skylab (19731974), Solwind (1979-1985) and Solar Maximum Mission (1980-1989). These early missions made good observations of individual events, but long-term surveys suffered from low sensitivity, small fields of view that didn't encompass the whole Sun, and low data cadence. The Solar and Heliospheric Observatory (SoHO), launched in 1995, began a systematic study of CMEs, which continues to the present. SoHO makes excellent observations of CMEs for events that originate near the limbs of the Sun. The image projected onto the sky shows clear structure and the radial expansion velocity can be readily measured. By contrast, CMEs that are headed towards Earth appear as 'halo CMEs', with little discernible 


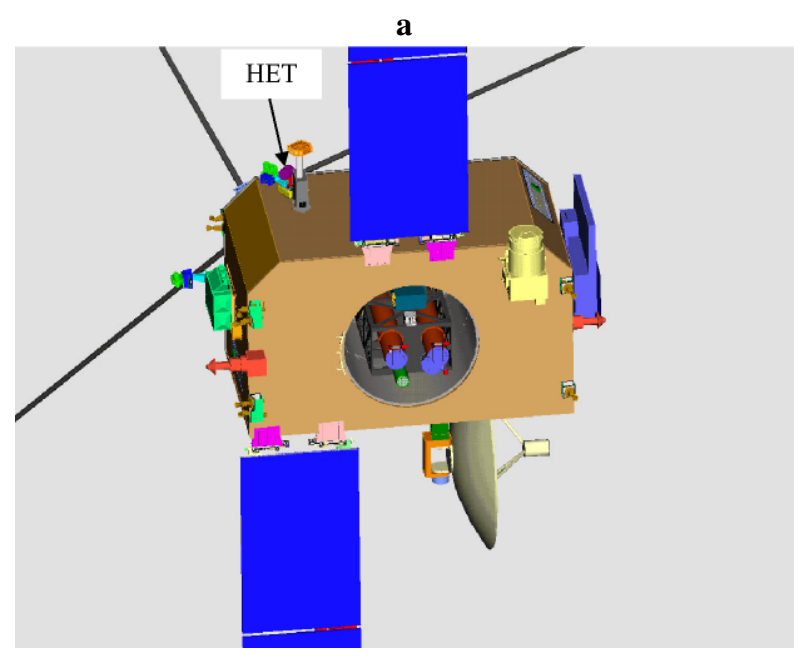

b

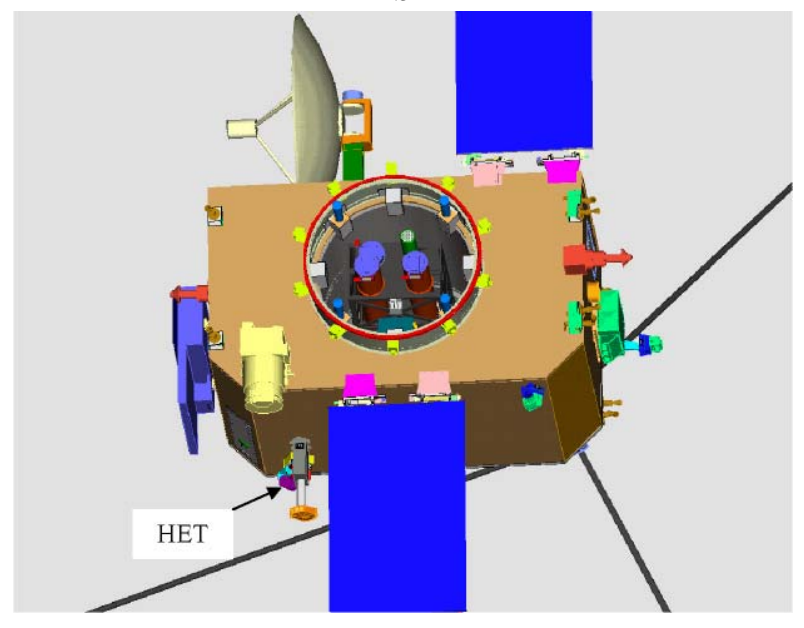

Fig. 4 a The Ahead spacecraft viewed from somewhat above the ecliptic plane (SEP Central, with HET and LET mounted on top, is on the top of the spacecraft to the left and behind the solar panel). $\mathbf{b}$ The Behind spacecraft viewed from somewhat below the ecliptic plane (SEP Central is mounted on the underneath side of the spacecraft). Note the deployed dish antennas looking towards Earth in each case (Earth is approximately midway between the two spacecraft). Both HETs look along the nominal Parker spiral towards the Sun

structure. In addition, it is very difficult to determine radial expansion velocities. Of course these are the events that have the most impact on Earth. STEREO observations, made from two spacecraft on opposite sides of the Earth-Sun line, will be able to circumvent many of these problems.

Interplanetary shock waves driven by CMEs have been observed in plasma and magnetic field data for a long time, and it has long been known that low energy particles (e.g., $<40 \mathrm{MeV}$ protons) are often seen in association with passing shocks (e.g., Bryant et al. 1962; Cohen 2006). The first set of spacecraft to be able to make long-term, multi-point observations of energetic particles at $\leq 1$ AU consisted of Helios 1 and 2 (launched into solar orbit 
Fig. 5 Shows proton intensities versus time for the SEP event of 4 November 2001 as observed by the NOAA/GOES satellites. The corresponding energies are provided in the figure

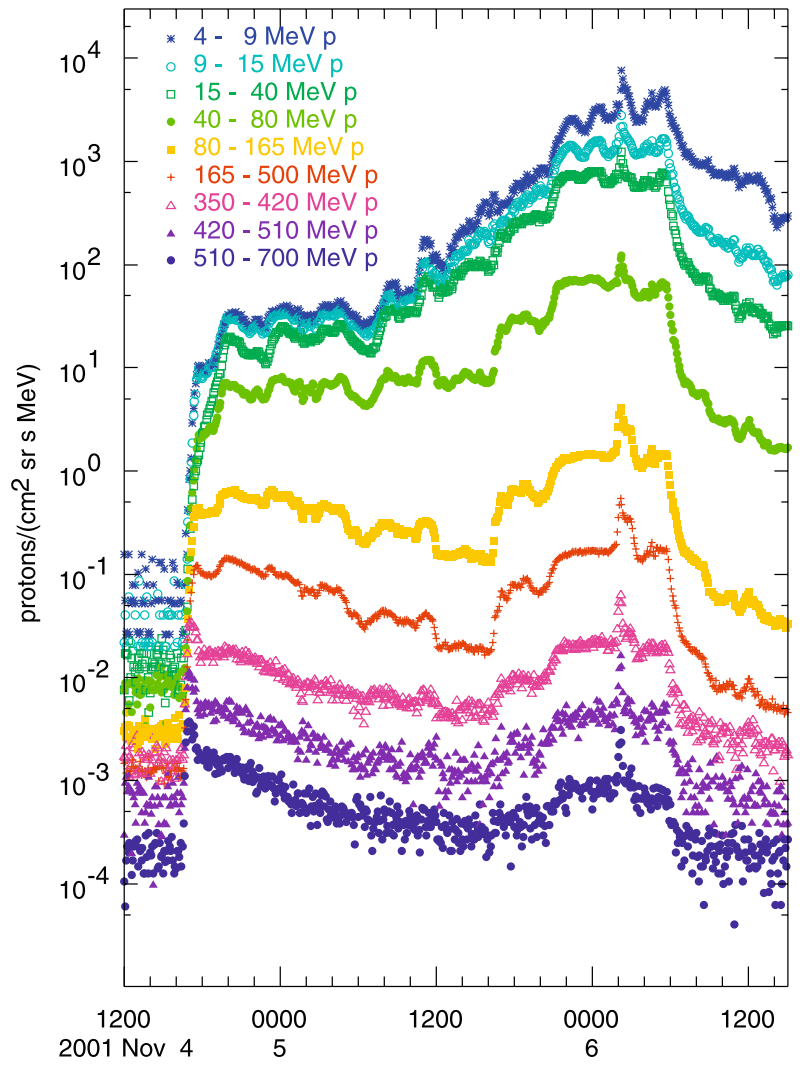

in 1974 and 1976) together with IMP-8 at Earth. Unfortunately, there were essentially no CME observations associated with particle events observed by these spacecraft. The advent of STEREO will dramatically change this situation.

Recent reviews of our understanding of solar energetic particles include Reames (1999) and von Rosenvinge and Cane (2006). Reames has made extensive arguments to support the idea that all large, CME-associated solar energetic particle (SEP) events are accelerated by the associated CME-driven shock. Although others have argued that some high energy particles originate in the associated flare, there can be no doubt that acceleration by the CMEdriven shock is a dominant effect at low energies and, on occasion, this effect is clearly observed at energies above $1 \mathrm{GeV}$. A striking example is shown in Fig. 5, which shows proton intensities for the event of 4 November 2001: there is a sharp peak at the shock (around 02:00 on 6 November 2001) at all energies shown, including the highest energy interval for which proton data is available, $510-700 \mathrm{MeV}$. It is also apparent that the increases shown for energies from $15 \mathrm{MeV}$ to $>350 \mathrm{MeV}$, starting at $\sim 17: 00$ on 5 November and ending at $\sim 07: 00$ on 6 November (a total period of 15 hours) are closely associated with the shock. No doubt all the particles seen in this event have this same origin.

Many other observations also emphasize the importance of shock acceleration in SEP events. For example, the observations of streaming-limited proton intensities in SEP events has been shown to originate with the generation of waves by protons in the vicinity of the shock (Reames and Ng 1998). Cohen et al. (2005) and Mewaldt et al. (2005) have reported on spectral breaks that they interpret as being due to rigidity-dependent escape from shocks 
Fig. 6a Example of simultaneous SEP observations at different solar longitudes. Note the substantial differences in intensity profiles even though the spacecraft are not widely separated in longitude. From Reames et al. (1997)

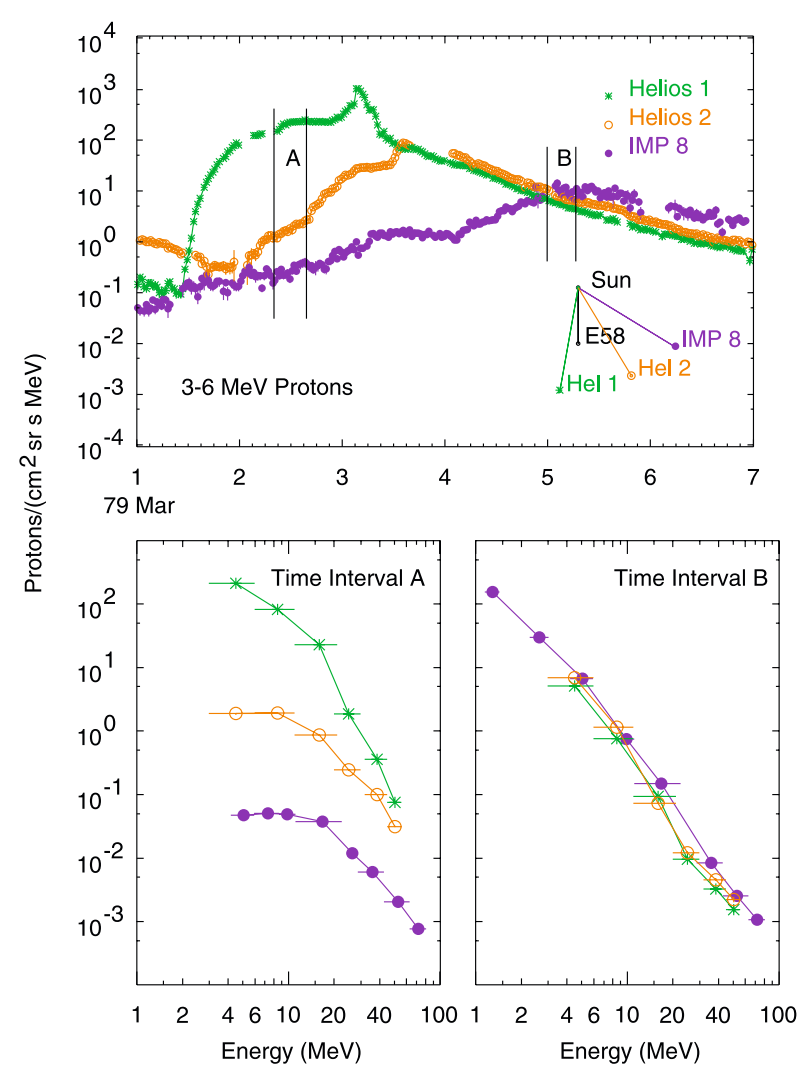

accelerating the observed particles. They find that $\mathrm{H}, \mathrm{He}$, and $\mathrm{O}$ spectra "resemble double power laws, with a break in the spectral index between $\sim 5$ and $\sim 50 \mathrm{MeV} /$ nucleon which appears to depend on the charge-to-mass ratio of the species." Since the HET has a strong overlap with these energies, HET will be vital for observing spectral breaks in SEP spectra.

Simultaneous observations of energetic particles at different solar longitudes are shown in Figs. 6a and 6b. These observations were made by Helios 1 and 2 and IMP-8 (Reames et al. 1997). In each figure there is an inset that shows the relative positions of the 3 spacecraft. The longitude of the corresponding active region is given by the black line in each figure. We see in the first figure substantial initial differences in intensity profiles even though the spacecraft are not widely separated in longitude. Somewhat more than 3 days after the first particles were seen, the decay profiles at all three spacecraft are essentially identical, suggesting that after that they are inside a region with fairly uniform and decaying particle intensity. We see much the same behavior in the second figure. The differences are (1) in the second figure, the spacecraft are separated considerably more in longitude, and (2) the initial differences are actually smaller in the second event than they were in the first. The different features in these two events are most likely associated with differences in the associated CMEs. With STEREO (and with WIND and ACE near Earth) we will have the opportunity to investigate such effects with a greatly expanded data set. If flare particles are sometimes a major contributor to SEP events, we might expect to see them more clearly at one spacecraft than another due to different connections being made to the flare region. Tylka and Lee (2006) have suggested that enhancements of heavy nuclei abundances in SEP events may 
Fig. 6b Another example of simultaneous SEP observations at different solar longitudes. Note how similar the intensity profiles are after $\sim$ mid-day of 25 September despite the large differences in solar longitude. From Reames et al. (1997)

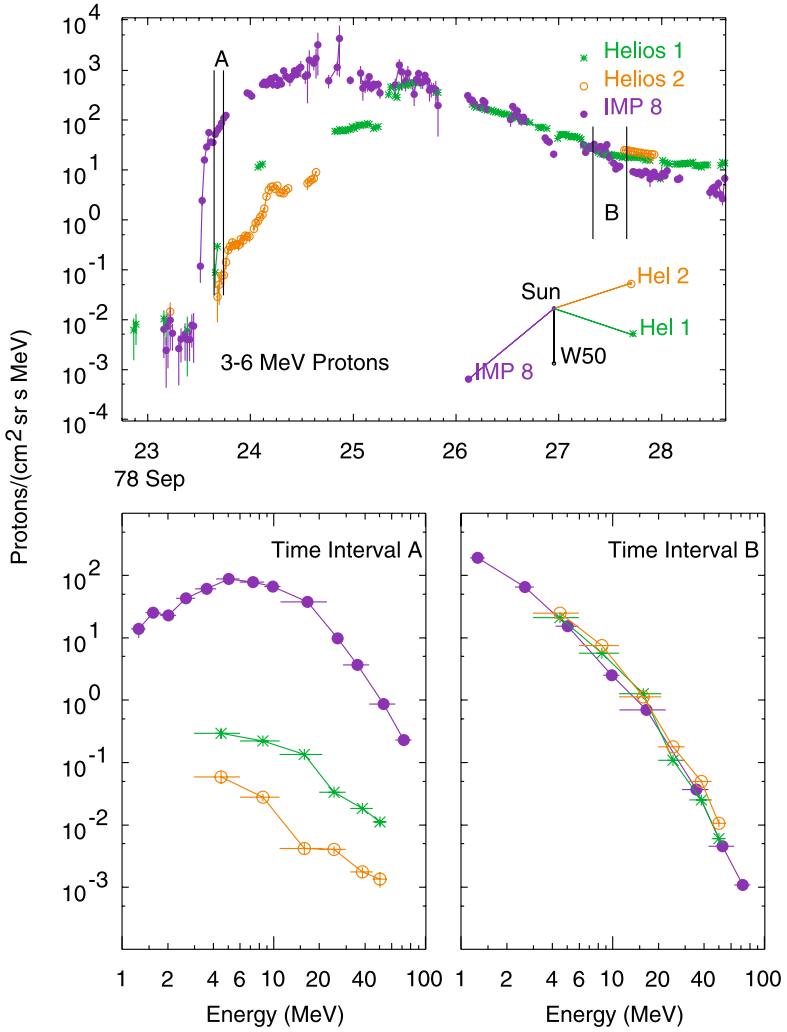

originate at the flanks of CMEs where the shocks are more likely to be quasi-perpendicular. This too might be discernible by multiple spacecraft as a longitudinal variation of observed abundances. At the time of the older missions like Helios 1 and 2, access to data from different instruments was fairly restricted. We now have the advantage that data from all the different instruments on STEREO are readily available.

Within the past two years NASA has decided to send astronauts back to the Moon for extended periods of time, with the goal of eventually sending humans to Mars and back. Although there has been a continuous human presence in space over the past decade on MIR and the International Space Station, these missions were carried out within the protective cover of Earth's magnetosphere, which shields against most galactic cosmic rays and solar energetic particles over much of the orbit. Once astronauts leave Earth orbit, they will be subject to increased radiation risks due to cosmic rays and large SEP events. The same applies to the electronic instrumentation that they will depend on. This has focused increased attention on the need to characterize the highly variable SEP radiation environment, with a goal of providing improved forecasts of the intensity and timing of the largest events. Spectral roll-overs will determine the severity of exposure to the most penetrating particles. The electrons observed by HET generally will arrive at earth before the ions by roughly 45 minutes, providing some advanced warning of impending high ion intensities. Warnings of the highest intensity ions, which occur at shocks, will be provided pre-shock by observing streaming-limited proton intensities (Reames 2001).

It takes a minimum of $\sim 10 \mathrm{MeV}$ for a proton to penetrate a space suit; for electrons the corresponding energy is $\sim 1 \mathrm{MeV}$. Inside a pressure vessel the minimum proton energy 
is increased to $\sim 30 \mathrm{MeV}$. Thus, the response of HET is well matched to monitoring SEP protons, $\mathrm{He}$, and electrons in the critical energy range where they pose a radiation risk to humans. The multi-point Beacon measurements (selected data broadcast to the ground continuously) provided by STEREO, supplemented by data from near-Earth spacecraft, will provide improved warning of the largest SEP events. In addition, STEREO studies using the full data set will lead to increased understanding of (1) which CMEs accelerate particles to high energies; (2) how SEPs are distributed in longitude; and (3) how interplanetary conditions govern particle transport in the inner heliosphere.

\subsection{Design Requirements}

The HET sensor must satisfy a number of design requirements to achieve the objectives in Sect. 2 and to support a range of studies that will be carried out by the SEP, IMPACT, and STEREO teams (see, e.g., Luhmann et al. 2007; Mewaldt et al. 2007b; Mason et al. 2007; and Müller-Mellin et al. 2007). These requirements were ultimately derived from the STEREO Missions Requirement Document, which states that the SEP suite shall measure the intensity, composition, energy spectra, and direction of energetic protons from 0.06 to 40 $\mathrm{MeV}$, heavier ions from $\sim 0.03$ to $40 \mathrm{MeV} / \mathrm{n}$, electrons from $\sim 0.03$ to $6 \mathrm{MeV}$, and ${ }^{3} \mathrm{He}$-rich solar particle events. The STEREO Level-1 Mission Requirements Document states that:

HET shall measure SEP ion fluxes, spectra, and composition covering the energy range from 30-80 MeV/n for $\mathrm{C}$ to Fe ions, 13 to $40 \mathrm{MeV/n}$ for $\mathrm{H}$ and $\mathrm{He}$, and 1 to $6 \mathrm{MeV}$ for electrons. The mass resolution shall be better than 0.3 amu for ${ }^{3} \mathrm{He}$ and

${ }^{4} \mathrm{He}$. Time resolution shall be 1 minute for $\mathrm{H}$ and $\mathrm{He}$ Beacon data, and 15 minutes otherwise. Shall handle at least 1000 events/sec.

The above statement has led to the requirements and goals summarized in Table 2, which were the basis for the detailed design of the HET. Table 2 also shows the capability of the two HET instruments, which meet or surpass all of the requirements.

\section{Instrument Description}

The HET telescope uses the classic $\Delta E \times E-\Delta E$ method of particle identification for particles that stop in the telescope. The method is extended to include particles that penetrate the telescope, thereby extending the observed energy range of protons and $\mathrm{He}$ in particular. Here $\Delta E$ refers to the energy loss of a particle with incident kinetic energy $E$ as it traverses a thin detector with thickness $\Delta x$, and $E-\Delta E$ (also denoted by $E^{\prime}$ ) is the residual energy after passing through the $\Delta E$ detector. In a plot of $\Delta E$ versus $E^{\prime}$, particles with different charge $Z$ and mass $A$ are separated into different tracks. The location on each track depends upon the incident energy. A sample such plot based upon a Monte Carlo simulation of HET is shown in Fig. 7. Note the separation of ${ }^{3} \mathrm{He}$ and ${ }^{4} \mathrm{He}$.

\subsection{HET Detectors}

The HET detectors are ion-implanted Si detectors provided by Micron Semiconductor, Ltd. in the UK. This type of detector is well known for its excellent linearity of energy loss response and ruggedness. The HET detectors are all nominally $1 \mathrm{~mm}$ thick and are operated with a bias voltage of 175 volts. Estimates of the detector thicknesses were obtained by averaging the thicknesses of wafer samples adjacent to where each detector was cut out from its wafer. For example, for FM1 these estimates ranged from 989-1015 $\mu \mathrm{m}$. 
Table 2 HET design requirements

\begin{tabular}{|c|c|c|c|}
\hline Description & Requirement & Goal & Capability \\
\hline Field of view & $\geq 50^{\circ}$ cone & $60^{\circ}$ cone & $55^{\circ}$ cone \\
\hline $\begin{array}{l}\text { Energy range } \\
(\mathrm{MeV} / \mathrm{n})\end{array}$ & $\begin{array}{l}\mathrm{H}, \mathrm{He}: 13-40 \\
{ }^{3} \mathrm{He}: 16-40 \\
6 \leq Z \leq 14: \sim 30-80\end{array}$ & $\begin{array}{l}\mathrm{H}, \mathrm{He}: 13-100 \\
{ }^{3} \mathrm{He}: 16-50 \\
6 \leq Z \leq 14: \sim 30-80\end{array}$ & $\begin{array}{l}\text { H, He: } 13-100 ; \\
{ }^{3} \mathrm{He}: 17-47 ; \\
Z=6: 27-74 \\
Z=26: 52-163\end{array}$ \\
\hline $\begin{array}{l}\text { Geometry factor } \\
\left(\mathrm{cm}^{2} \mathrm{sr}\right)\end{array}$ & $\begin{array}{l}\text { H, He: } 0.1-0.5 \\
6 \leq \mathrm{Z} \leq 26: 0.5\end{array}$ & $\begin{array}{l}\mathrm{H}, \mathrm{He}: 0.15-0.7 \\
6 \leq \mathrm{Z} \leq 26: 0.7\end{array}$ & $\begin{array}{l}\text { H, He: } 0.10-0.61 \\
6 \leq Z \leq 26: 0.61\end{array}$ \\
\hline $\begin{array}{l}\text { H1 detector noise } \\
\text { level }\end{array}$ & $<40 \mathrm{keV} \mathrm{rms}$ & $\leq 30 \mathrm{keV} \mathrm{rms}$ & $<30 \mathrm{keV} \mathrm{rms}$ \\
\hline Element resolution & $\begin{array}{l}\delta Z \leq 0.2 \text { charge units } \\
\text { for } 1 \leq Z \leq 14\end{array}$ & $\begin{array}{l}\delta Z \leq 0.3 \text { charge units } \\
\text { for } 16 \leq Z \leq 26\end{array}$ & $\begin{array}{l}\delta Z \leq 0.2 \text { for } 1 \leq Z \leq 14 \\
\delta Z \leq 0.3 \text { for } 16 \leq Z \leq 26\end{array}$ \\
\hline $\begin{array}{l}{ }^{4} \mathrm{He} \text { mass } \\
\text { resolution }\end{array}$ & $\leq 0.3 \mathrm{amu} \mathrm{rms}$ & $\leq 0.2 \mathrm{amu} \mathrm{rms}$ & $\leq 0.2 \mathrm{amu} \mathrm{rms}$ \\
\hline $\begin{array}{l}\text { Maximum event } \\
\text { rate }\end{array}$ & 1000 per sec & 5000 per sec & $\sim 4000$ per sec \\
\hline Energy binning & 6 intervals per species & $\begin{array}{l}8 \text { intervals per } \\
\text { species }\end{array}$ & $\begin{array}{l}10 \text { intervals per species } \\
\text { for } H \text { and } Z \geq 2\end{array}$ \\
\hline $\begin{array}{l}\text { Onboard species } \\
\text { identification }\end{array}$ & $\begin{array}{l}\mathrm{H},{ }^{3} \mathrm{He},{ }^{4} \mathrm{He} \text {, electrons, } \\
6 \leq Z \leq 14\end{array}$ & $\begin{array}{l}\text { Also identify } \\
16 \leq Z \leq 26\end{array}$ & $\begin{array}{l}\mathrm{H},{ }^{3} \mathrm{He},{ }^{4} \mathrm{He} \mathrm{C}, \mathrm{O}, \mathrm{Ne} \\
\mathrm{Mg}, \mathrm{Si}, \mathrm{Fe}, \text { electrons }\end{array}$ \\
\hline Time resolution & $\begin{array}{l}15 \mathrm{~min} \text {; Telemeter } 0.3 \\
\text { prioritized event/sec }\end{array}$ & $\begin{array}{l}15 \mathrm{~min} \text {; Telemeter } 1 \\
\text { prioritized event/sec }\end{array}$ & $\begin{array}{l}15 \mathrm{~min} \text {; Telemeter } \sim 2 \\
\text { prioritized events/sec }\end{array}$ \\
\hline Beacon telemetry & $\begin{array}{l}1 \mathrm{~min} \text { for } \mathrm{H}, \mathrm{He} \text {, } \\
\text { electrons }\end{array}$ & $\begin{array}{l}1 \mathrm{~min} \text { for } \mathrm{H}, \mathrm{He} \text {, } \\
\text { electrons }\end{array}$ & $\begin{array}{l}\text { 1-min for } \mathrm{H}, \mathrm{He}, \mathrm{C}, \mathrm{O} \\
\mathrm{Fe} \text {, electrons }\end{array}$ \\
\hline
\end{tabular}

\subsubsection{H1, H2 Detectors}

The $\mathrm{H} 1$ detectors were made using planar, polygonal Si disks, $1 \mathrm{~mm}$ thick, that are mounted in a mechanical mount constructed using printed circuit board/flex-circuit technology. Figure $8 \mathrm{a}$ is a picture of the junction side of an $\mathrm{H} 1$ detector; Fig. $8 \mathrm{~b}$ is a picture of the ohmic side. Gold-plated copper pads on the mount are used to make redundant wire-bond connections between the detector electrode surfaces and copper traces that run through the mount and flex-circuit to a connector that plugs into the flight electronics board. The H1 detector has two separate sensitive areas on the junction side that are defined by ion implanting 2 separate $\mathrm{p}-\mathrm{n}$ junctions. The inner sensitive area is a circle, $0.8 \mathrm{~cm}$ in diameter. The outer sensitive area is a concentric ring, with outer diameter $2 \mathrm{~cm}$, around the inner circular area. The outer area is separated from the inner area by a circular gap that is $60 \mu \mathrm{m}$ wide. In addition, the outer ring has a narrow (130 $\mu \mathrm{m}$ wide) radial area cut-out through which passes a narrow extension of the central area that terminates in a small pad (one $\mathrm{mm}$ by $0.28 \mathrm{~mm}$ ) located 
Fig. 7 Simulated response of HET telescope to incident electrons, hydrogen (protons), ${ }^{3} \mathrm{He},{ }^{4} \mathrm{He}, \mathrm{C}, \mathrm{O}, \mathrm{Ne}, \mathrm{Mg}, \mathrm{Si}$, and $\mathrm{Fe}$. Electrons are not well-modeled in this figure
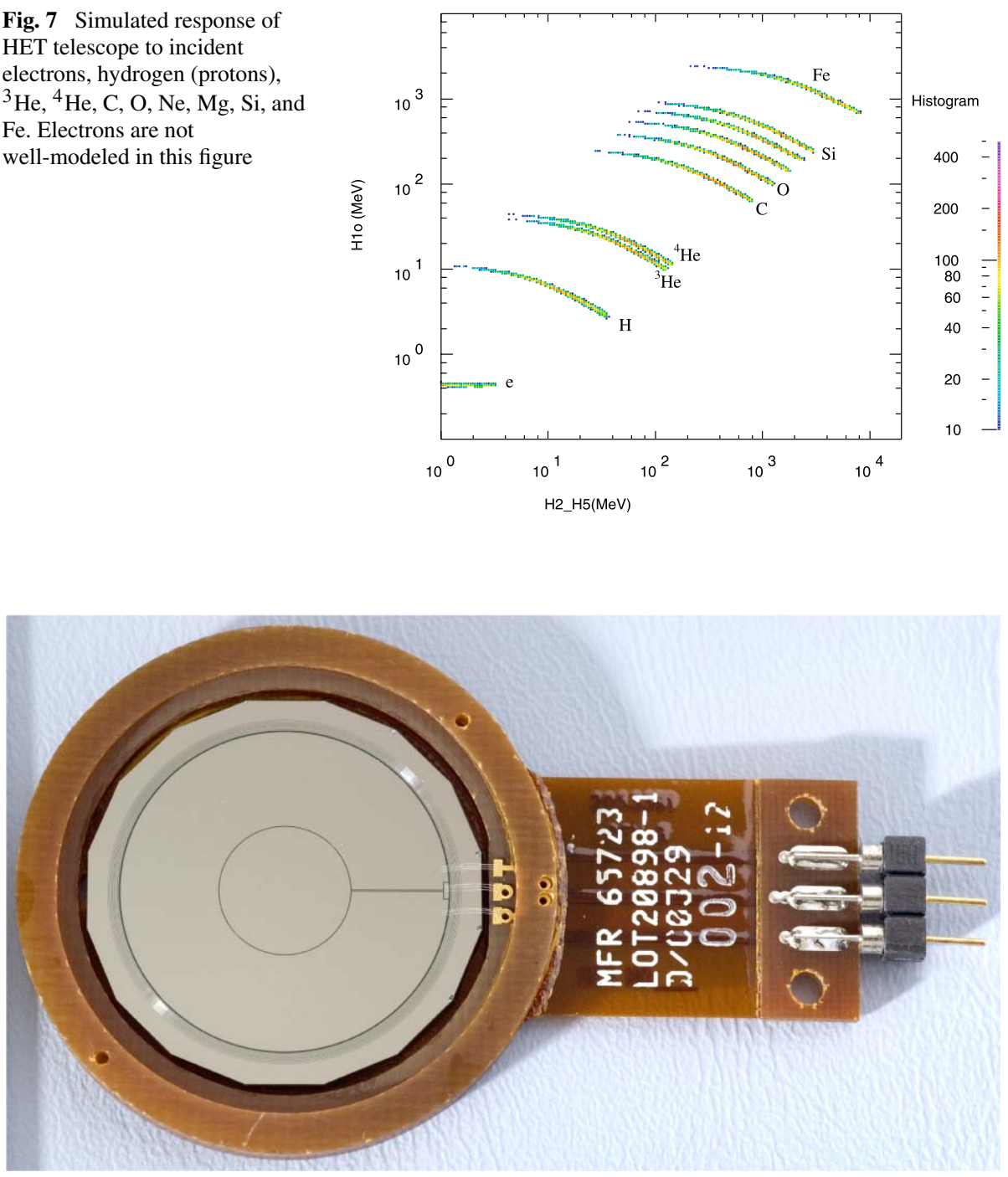

Fig. 8a Shows the junction side of an $\mathrm{H} 1$ detector

just inside of the outer circular ring. There is only a single, circular charge collection surface on the ohmic side. Surrounding the outer sensitive area on the junction side is a stepped guard intended to reduce excess detector leakage current. The stepped guard consists of a sequence of separate, narrow metalized rings that are allowed to float electrically.

In effect, the $\mathrm{H} 1$ detector consists of two detectors, one inner circular detector and one outer ring detector. These are designated $\mathrm{H} 1 \mathrm{i}$ and $\mathrm{H} 1 \mathrm{o}$, respectively.

The $\mathrm{H} 2$ detector is identical to the $\mathrm{H} 1$ detector but it has the two separate areas on the junction side electrically shorted together external to the detector mount. In effect, the $\mathrm{H} 2$ detector acts as a circular detector $2 \mathrm{~cm}$ in diameter by $1 \mathrm{~mm}$ thick. 


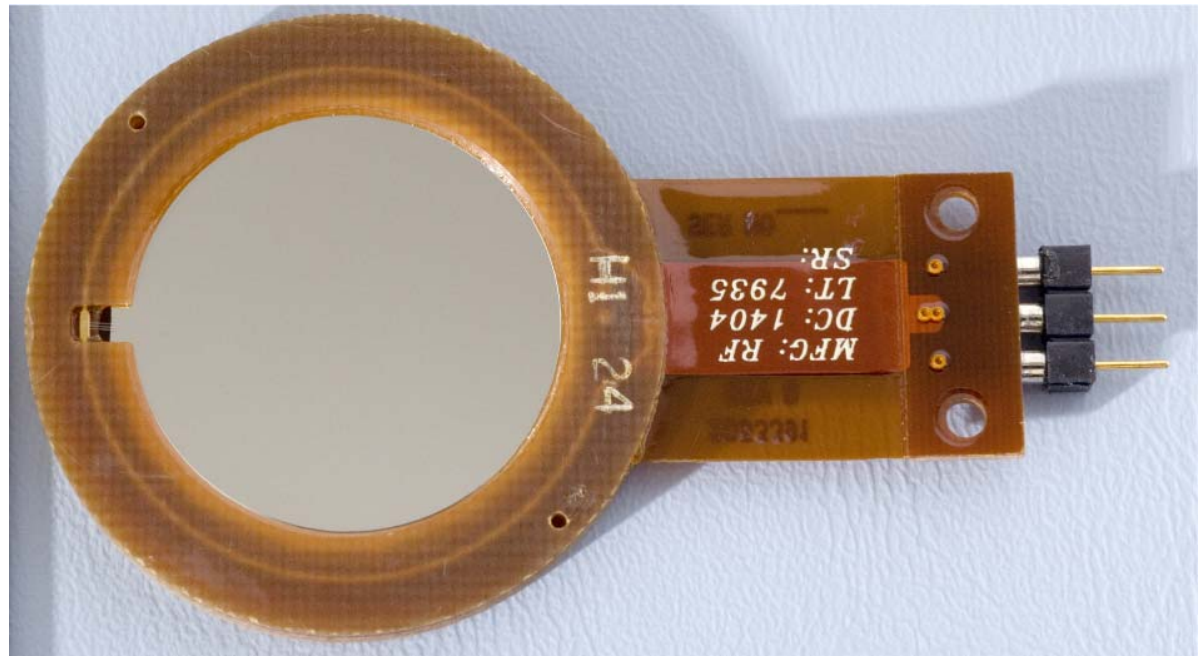

Fig. 8b Shows the ohmic side of an $\mathrm{H} 1$ detector

\subsubsection{HET Stack Detectors}

The remainder of the HET telescope is constructed using a second detector type that is similar to the $\mathrm{H} 1$ detector except that it only has a single circular $\mathrm{p}-\mathrm{n}$ junction with diameter $4 \mathrm{~cm}$. One of these detectors, which we will refer to as a stack detector, is shown in Fig. 9.

\subsubsection{Detector Testing}

Detector quality checking was performed at three different locations: initially Micron Semiconductor, Ltd. did thermal cycling at ambient pressure, thickness measurements on wafer samples, and vibration testing prior to shipment of the detectors to Caltech. Caltech inspected the detectors for scratches and photographed the bond wires prior to shipment to GSFC. GSFC then made the following measurements:

1. Detector leakage current versus bias voltage at ambient pressure and temperature with a maximum test bias voltage of $250 \mathrm{~V}$.

2. Detector capacitance versus bias voltage at ambient pressure and temperature.

3. ${ }^{241} \mathrm{Am}$ alpha particle exposures in vacuum through both the junction and ohmic detector sides at different bias voltages, including the flight operating bias of $175 \mathrm{~V}$. We positioned the uncollimated source at 1.5 detector diameters from the center of the detector and looked for the alpha peak to be at $\sim 5.48 \mathrm{MeV}$ with similar width for both front and back exposures. In at least one case the flight detector $(\mathrm{H} 2$ on the Behind spacecraft) did not quite live up to this requirement, but the schedule didn't permit a late substitution.

4. So-called lifetest, where up to 20 detectors were operated at $+40^{\circ} \mathrm{C}$ over an extended period in vacuum (a few times $10^{-6}$ Torr or less), typically 3 weeks, with a single cycle of $\sim$ two days to $-25^{\circ} \mathrm{C}$. Detector leakage current and noise were monitored continuously during lifetest.

Sample test results for test types 1 and 2 are shown in Figs. 10 and 11. The intent of test 1 is to look for evidence that a detector might be operating too close to reverse bias breakdown. 


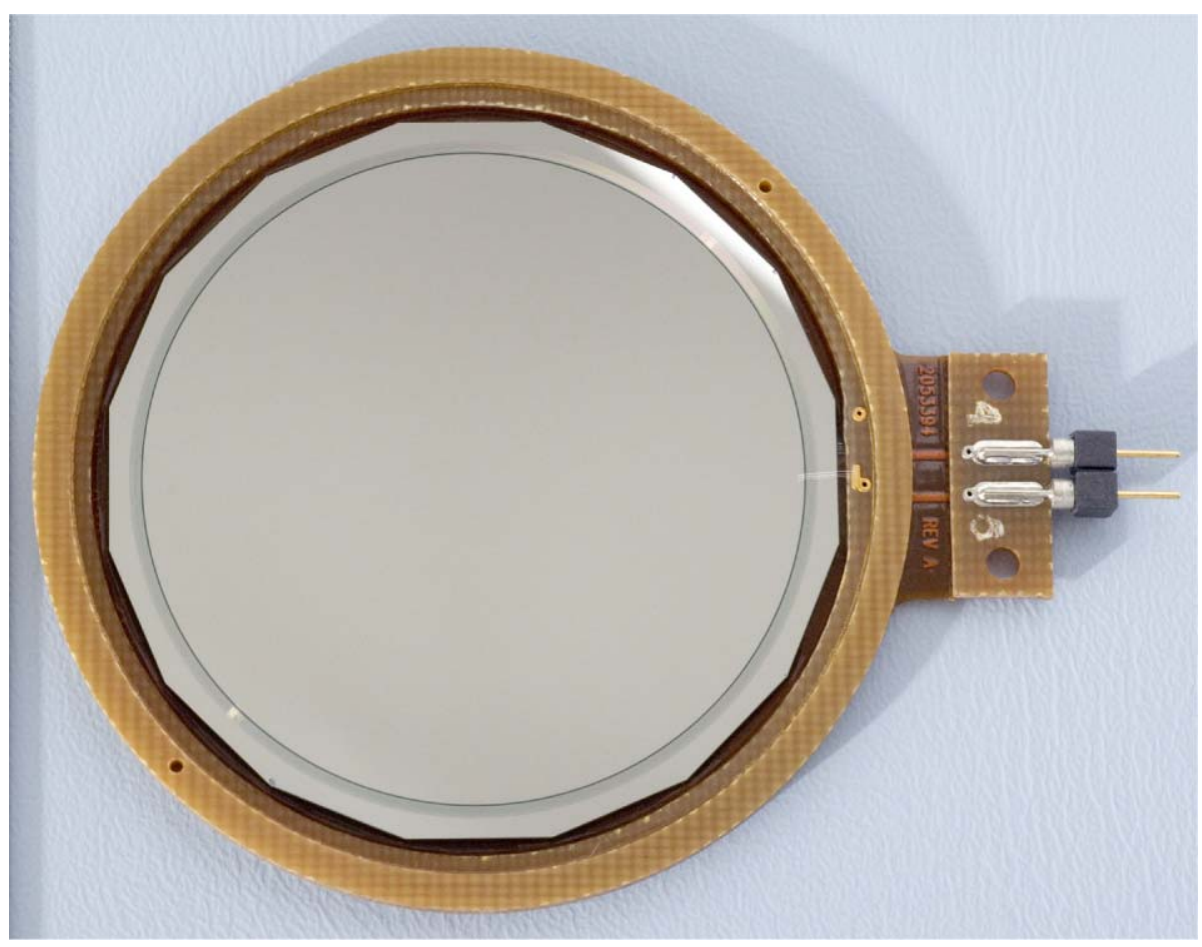

Fig. 9 Shows a HET stack detector (junction side)

Fig. 10 Shows detector leakage current versus bias voltage for a sample $\mathrm{H} 3$ detector

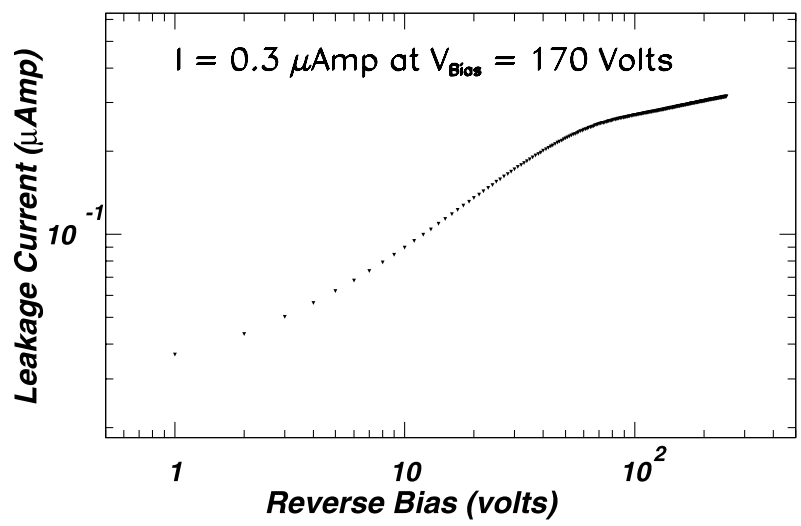

The voltage above which the capacitance saturates in test type 2 gives an indication of the voltages at which the detectors are fully-depleted. Test type 3 gives a separate measure of detector depletion, and in general full-depletion is only observed at higher voltages than are indicated by test type 2 .

We initially received detectors that had a double passivation layer that was intended to make the detectors more resistant to attack by chemical vapors in the air. We observed that most of the initial detectors might behave well for awhile in lifetest, but eventually their leakage currents would start to grow without check. In the end we flew only detectors with a 
Fig. 11 Shows the detector capacitance versus bias voltage curve for a sample $\mathrm{H} 3$ detector

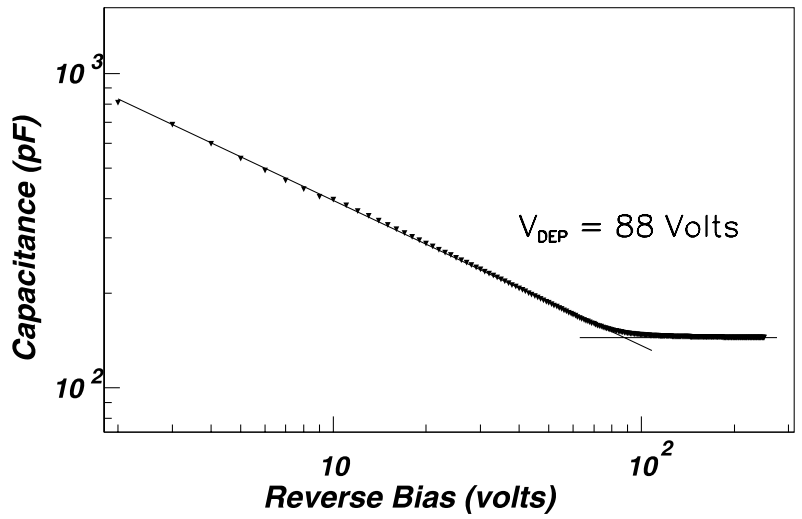

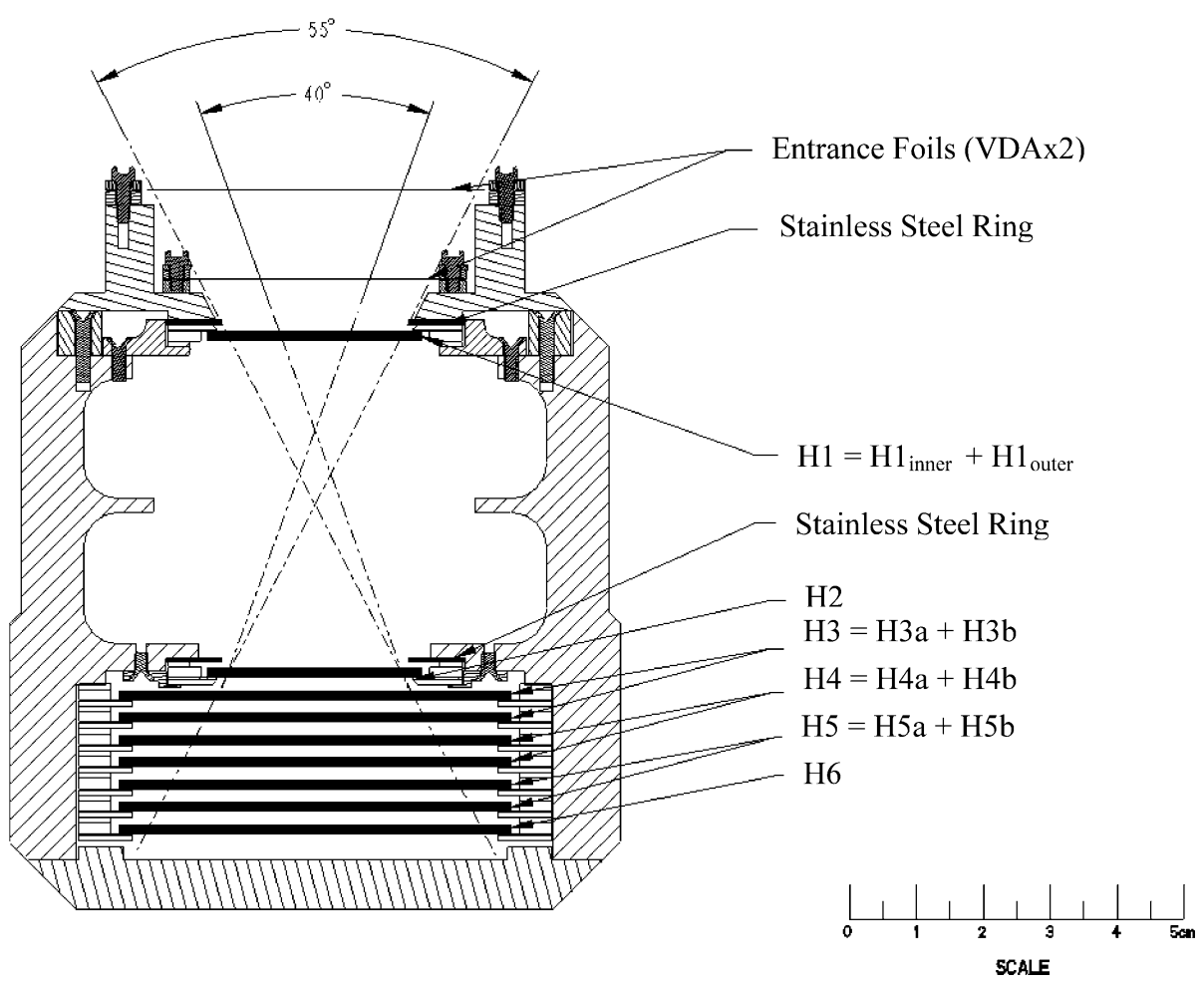

Fig. 12 Shows a schematic cross-section of a HET telescope

single passivation layer. These detectors rarely had problems with steadily growing leakage currents, and the few that did have such problems were rejected.

\subsection{HET Telescope}

Figure 12 shows a cross-sectional view of an assembled HET telescope. The spacing between the bottom of the $\mathrm{H} 1$ detector and the top of the $\mathrm{H} 2$ detector is $3.68 \mathrm{~cm}$. The spacing 
between the remaining detectors is nominally $0.15 \mathrm{~cm}$. Note that the outputs of detectors $\mathrm{H} 3 \mathrm{a}$ and $\mathrm{H} 3 \mathrm{~b}$ are summed into a single pulse height analyzer. This detector pair is referred to as H3. Similarly, $\mathrm{H} 4$ and $\mathrm{H} 5$ each consist of 2 detectors summed together. The acceptance angles for particles stopping in the telescope are defined by H1o and H1i and H2. H6 is a single detector that determines whether or not a particle stops in the preceding detectors: 'stopping particles' enter the telescope through the H1 detector but don't reach the H6 detector; 'penetrating particles' traverse $\mathrm{H} 1$ and reach H6. Most penetrating particles penetrate H6 as well.

The telescope body is designed to prevent external light from reaching any detector surface. The front end of the telescope is blocked by two separate Kapton foils, each $0.005^{\prime \prime}$ thick and coated front and back with vapor deposited aluminum (VDA). The double foil design (as contrasted with a single foil of double the thickness) is intended to decrease the effects of pin-holes in the VDA and also to provide better protection from incident micrometeors. The body of the telescope is also designed to reduce the numbers of low energy particles that can reach any of detectors $\mathrm{H} 2$ through $\mathrm{H} 6$ without passing through $\mathrm{H} 1$ first. In particular, $0.5 \mathrm{~mm}$ thick stainless steel rings with inner diameter of $21.0 \mathrm{~mm}$ are included in the body in front of $\mathrm{H} 1$ and $\mathrm{H} 2$ (see Fig. 12). Further details of the mechanical and thermal design of the combined LET/HET/SEP Central package may be found in Mewaldt et al. (2007b).

\subsection{HET Electronics}

\subsubsection{Introduction}

Figure 2 shows a block diagram of the IMPACT Solar Energetic Particles suite, including the HET and SEP Central. SEP Central includes a low-voltage power supply for all of IMPACT/SEP, a bias supply for all the solid-state detectors, EEPROM for storing on-board code, response tables, and boot-up command states, and a central Minimal Instruction Set Computer (MISC). The SEP Central MISC performs the following functions:

1. It receives commands from the IMPACT IDPU (see Luhmann et al. 2007) and forwards them to the appropriate subsystem (in our case, HET); it also receives command echoes from each subsystem and assembles them into telemetry packets, which are then passed back to the IDPU.

2. It time-tags HET telemetry packets with Universal Time and transmits them to the IDPU.

3. It assembles housekeeping data and so-called Beacon data from each subsystem into telemetry packets, which it then sends to the IDPU.

4. It receives and stores in EEPROM the on-board code and tables for LET and HET as well as for SEPT, SIT, and the SEP Central MISC. It also transmits this information to the appropriate subsystem when the latter is booted up.

SEP Central is described more fully in the LET paper in the present volume (Mewaldt et al. 2007b). Note that, due to the central low-voltage power supply, all of SEP is either turned ON or OFF. For example, HET cannot be powered down without powering down all of the rest of SEP. Similarly, a common bias voltage (175 volts) is used for the $1 \mathrm{~mm}$ thick detectors in HET and LET.

Figure 13 shows a block diagram of the HET. The HET electronics board is housed in the same enclosure as SEP Central. It consists of a single printed circuit board with two PHASICs (Pulse Height Analysis System Integrated Circuits) supplied by Caltech, an ACTEL FPGA (Field Programmable Gate Array, part number RT54SX72S), three SRAM 


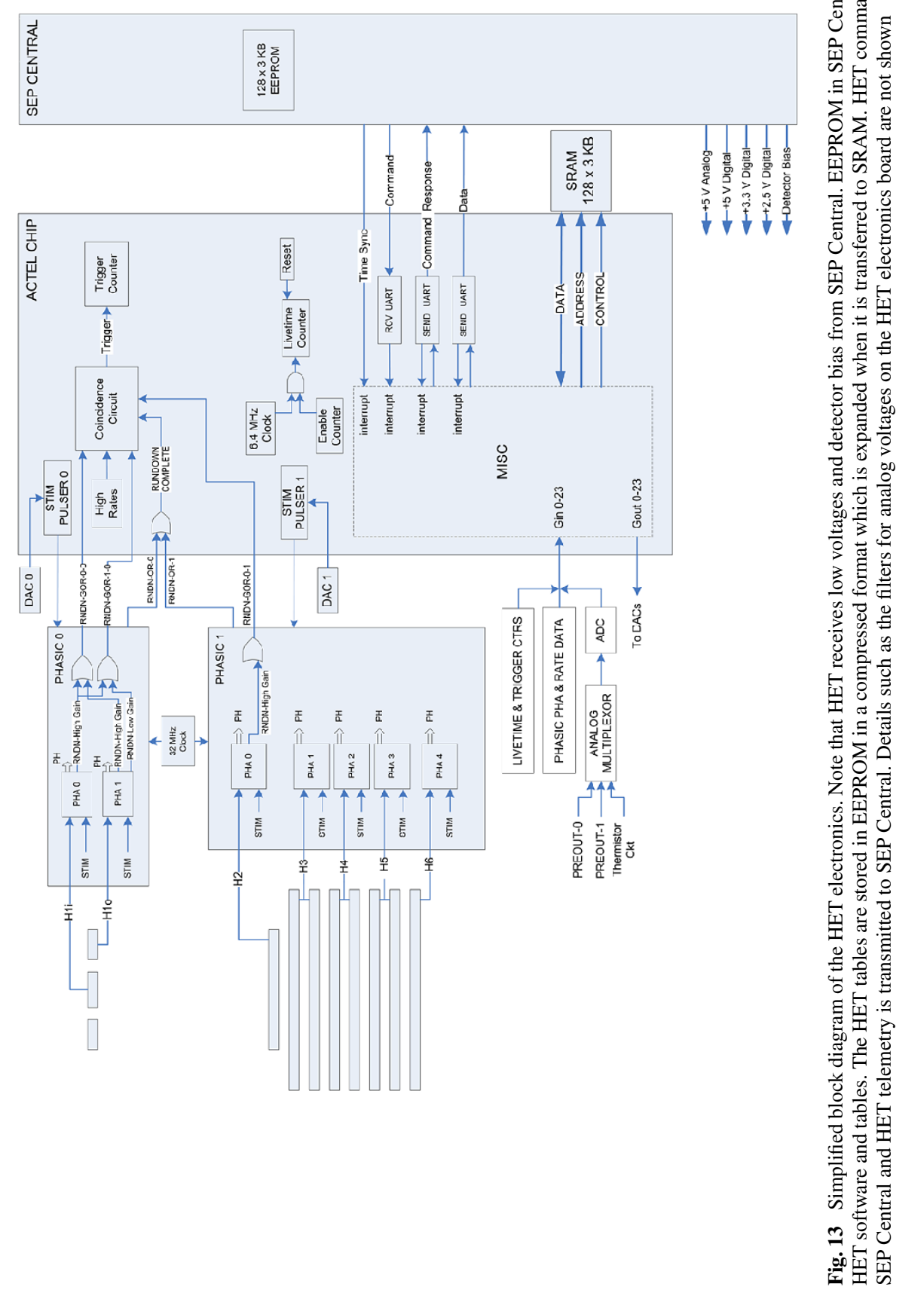


chips, and power-conditioning filters. The SRAM chips (Honeywell HLX6228) each have $128 \mathrm{~K} \times 8$ bits, for a total of $128 \mathrm{~K} \times 24$ bits for program and data storage.

Additional circuitry is provided for on-board pulsers that can stimulate each PHA channel, and to measure the leakage currents of each detector (or, in the case of $\mathrm{H} 3, \mathrm{H} 4$, and $\mathrm{H} 5$, pairs of detectors). Pulse height events that are created by the on-board pulsers will be referred to as "stim events".

The HETs and LETs are mounted differently with respect to SEP Central on each of the two STEREO spacecraft. This was shown earlier in Fig. 4. A single HET electronics board design permits the HET telescope to be mounted in either one orientation for the Ahead spacecraft or at $90^{\circ}$ to this orientation for the Behind spacecraft. The resultant look-direction for each HET in flight is towards the Sun along the nominal Parker spiral interplanetary magnetic field. Figure 14 shows pictures of a HET and its electronics board.

\subsubsection{PHASICs}

Charge pulses due to particles losing energy in the HET detectors are digitized by pulse height analyzers contained in an integrated circuit known as a PHASIC, designed by Rick Cook of Caltech (Cook 2002). A detailed description of the PHASIC is provided in Mewaldt et al. (2007b), so only a brief description will be provided here. A PHASIC contains 16 separate, large-dynamic-range pulse height analyzers (PHAs). Each of these separate PHAs has a charge-sensitive preamplifier with commandable gain. Each preamplifier output signal is coupled to two shaping amplifier/offset-gate/peak-detector/Wilkinson-ADC chains that operate in parallel, but with gains that differ by a factor of 20 . A variety of parameters of each separate PHA can be set by sending a single 108-byte serial command to the PHASIC. For example, individual unused PHA channels in a PHASIC can be powered down, the highgain and low-gain sections of each PHA can be disabled, and various test points internal to the chip can be steered to an output pin to be examined by an oscilloscope. Two different types of data may be extracted from the PHASIC: pulse height data outputs and the number of times high or low gain thresholds of each PHA have been triggered since reset (rate counter outputs). In flight we sample the rate counter outputs for each PHA channel every minute. A count is extracted as a 24-bit word. The pulse height data are also read out as 24 bits (we shall call this a raw pulse height). A raw pulse height includes the chip address, PHA address, gain, and overflow bits as well as the pulse height value. In addition, there are several read-out modes for pulse heights: in terse readout mode, which is the dominant mode used for flight, only the high gain pulse height is read out for each PHA that triggered unless the high-gain pulse height counter overflowed (i.e. went over 2047 counts); in the latter case only the low-gain pulse height is read out. The other readout mode is called the verbose mode. In this mode, both the high and low gain pulse heights are read out if both were triggered. The verbose mode is primarily for engineering diagnostics, however we also use it during high-rate mode (described later). Each PHA channel can be independently set to either terse or to verbose mode.

The format of PHASIC pulse height readouts is described in Table 3.

The token bit is set to 1 for the last pulse height read in a serial sequence of pulse heights being read out of the PHASICs. The stim bit has to be set by the MISC.

Note that HET uses two PHASICs. There are in principle more than enough PHA channels in a single PHASIC to service all the detectors in a HET. However, at the time of the initial telescope design, the first PHASICs were not available. There was some concern that there might be cross-talk within a single PHASIC, which could create artificial coincidences between the $\mathrm{H} 1$ and $\mathrm{H} 2$ detector channels. Two PHASICs were used to circumvent that possibility. 
Fig. 14 a Photograph of a HET telescope mated to its electronics board. The white cable is a 'connector-saver'. b Photograph showing the other side of the HET electronics board $\mathbf{a}$

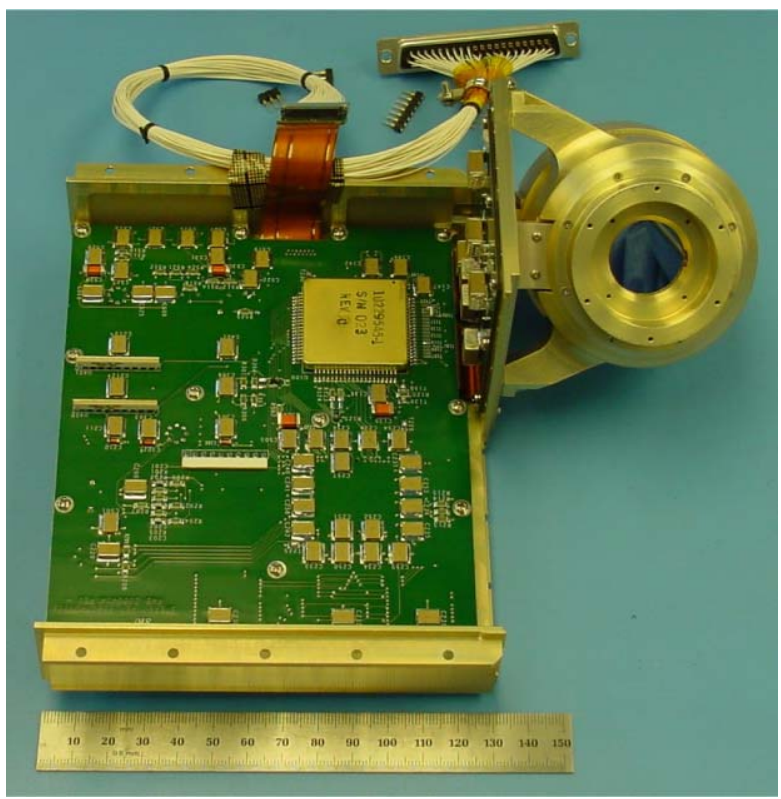

b

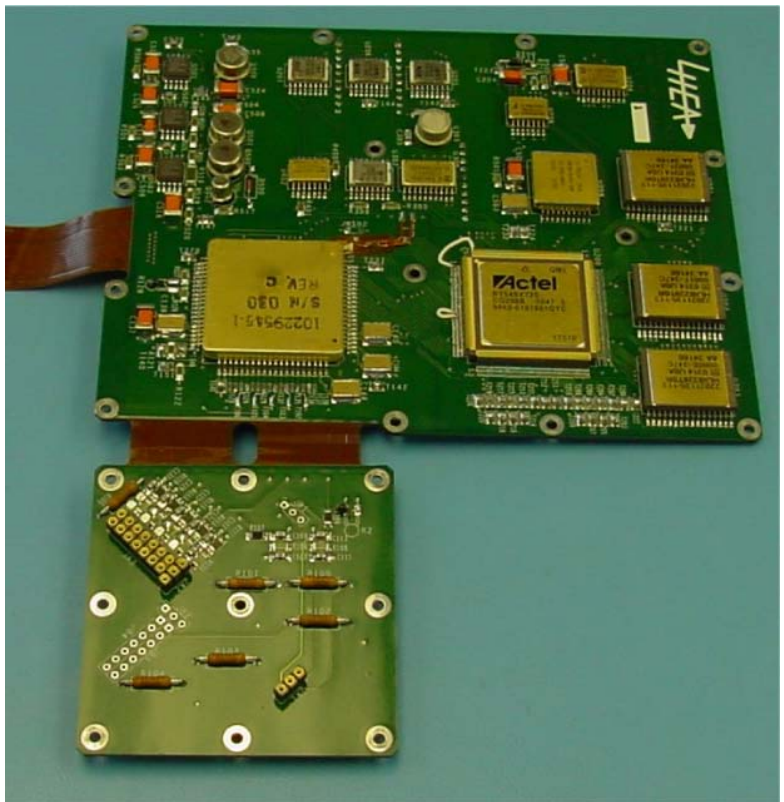

The dynamic range of the PHASICs is very large $(\sim 10,000)$, but the needs of the HET are somewhat larger. GEANT simulations of electrons (e.g., see Fig. 15) showed that, in order for minimum-ionizing electrons to remain above threshold in the $\mathrm{H} 1, \mathrm{H} 2$, and $\mathrm{H} 6$ detectors, the corresponding electronic thresholds needed to be set at no higher than $0.2 \mathrm{MeV}$. 
Table 3 Raw event format (23 bits)

\begin{tabular}{llllllll}
\hline 23 & $22-20$ & 19 & $18-15$ & 14 & $13-12$ & 11 & $10-0$ \\
\hline $\begin{array}{l}\text { Token } \\
\text { bit }\end{array}$ & Chip & Stim & PHA & High & Reserved & Overflow & Pulse \\
address & bit & address & gain bit & & bit & height \\
\hline
\end{tabular}

Fig. 15 GEANT 3.21

Simulation of $1 \mathrm{~mm}$ and $2 \mathrm{~mm}$ HET detector responses to minimum ionizing electrons at normal incidence

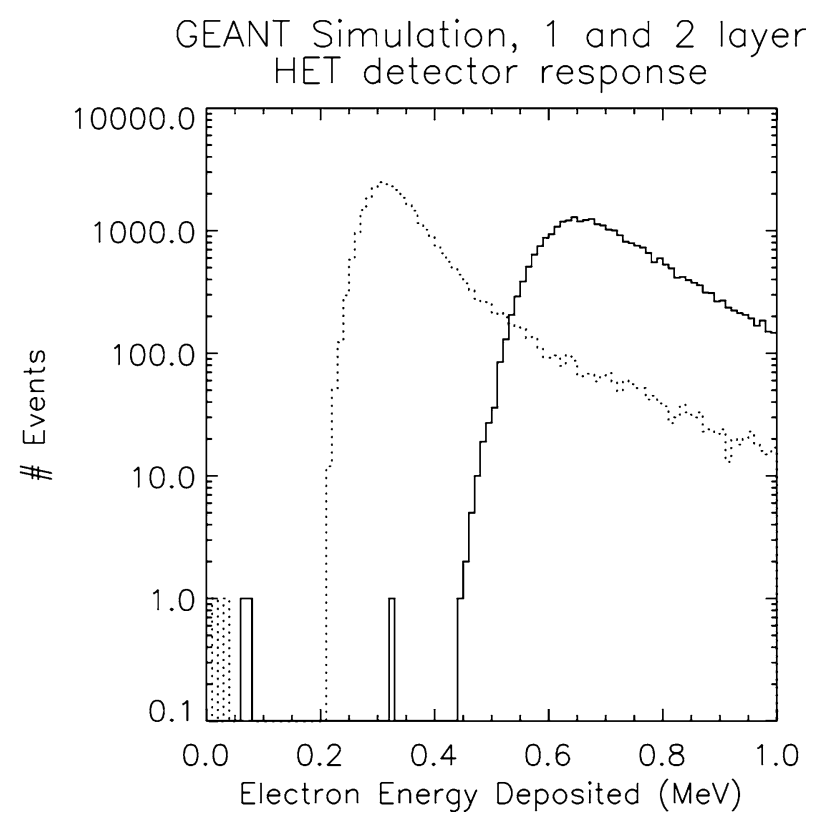

An Fe nucleus incident at an angle of $30^{\circ}$ from the normal to an $\mathrm{H} 1$ detector can lose as much as about $2800 \mathrm{MeV}$, leading to a total dynamic range of 2800/0.2 14,000. Since Fe is by far the most abundant element in SEPs above $\mathrm{Ca}(Z=20)$, we have limited Fe pulse heights to a maximum value (e.g., see Fig. 19a).

Finally, we note that for each PHA channel in a PHASIC, the thresholds for both high and low gain pulses are adjustable by command up to $\sim 6 \%$ of full-scale. This feature is not entirely intuitive because the PHA offset channel also changes when the threshold is changed. By offset channel, we mean the PHA channel versus input energy curve extrapolated to $0 \mathrm{MeV}$. Because pulse heights are offset-corrected on-board, it is essential that the offset channel stored on-board is changed at the same time that any change is made in the corresponding threshold. This is facilitated by changing the threshold setting, noting the shift in an on-board pulser peak, and then changing the offset to restore the peak channel position. The threshold values and offset channels are routinely reported in telemetry.

Tables $4 \mathrm{a}$ and $4 \mathrm{~b}$ provide the key parameters for each detector channel for the HETs onboard the Ahead (Table 4a) and Behind (Table 4b) spacecraft as they were configured at launch.

\subsubsection{Cross-Talk}

Cross-talk occurs when a large signal in one signal channel couples into another, producing an unintended signal in the second channel. During the first of two visits to the National Su- 
Table 4a PHASIC parameters for the HET on the Ahead spacecraft

Table 4b PHASIC parameters for the HET on the Behind spacecraft

\begin{tabular}{|c|c|c|c|c|}
\hline $\begin{array}{l}\text { Detector } \\
\text { channel }\end{array}$ & Gain & $\begin{array}{l}\text { Threshold } \\
(\mathrm{MeV})\end{array}$ & $\begin{array}{l}\text { Offset } \\
\text { channel }\end{array}$ & $\mathrm{MeV} /$ channel \\
\hline \multirow[t]{2}{*}{$\mathrm{H} 1 \mathrm{i}$} & High & 0.21 & 47.8 & 0.0524 \\
\hline & Low & 4.0 & 50.1 & 1.063 \\
\hline \multirow[t]{2}{*}{ H1o } & High & 0.2 & 47.4 & 0.0531 \\
\hline & Low & 21.0 & 33.0 & 1.066 \\
\hline \multirow[t]{2}{*}{$\mathrm{H} 2$} & High & 0.19 & 47.4 & 0.0524 \\
\hline & Low & 4.0 & 48.0 & 1.072 \\
\hline \multirow[t]{2}{*}{ H3 } & High & 0.38 & 46.7 & 0.1043 \\
\hline & Low & 9.0 & 47.3 & 2.087 \\
\hline \multirow[t]{2}{*}{$\mathrm{H} 4$} & High & 0.34 & 47.0 & 0.1044 \\
\hline & Low & 9.0 & 47.7 & 2.111 \\
\hline \multirow[t]{2}{*}{ H5 } & High & 0.34 & 46.7 & 0.1043 \\
\hline & Low & 9.0 & 47.7 & 2.099 \\
\hline \multirow[t]{2}{*}{ H6 } & High & 0.2 & 47.1 & 0.0520 \\
\hline & Low & 4.0 & 47.9 & 1.057 \\
\hline
\end{tabular}

\begin{tabular}{|c|c|c|c|c|}
\hline $\begin{array}{l}\text { Detector } \\
\text { channel }\end{array}$ & Gain & $\begin{array}{l}\text { Threshold } \\
(\mathrm{MeV})\end{array}$ & $\begin{array}{l}\text { Offset } \\
\text { channel }\end{array}$ & $\mathrm{MeV} /$ channel \\
\hline \multirow[t]{2}{*}{$\mathrm{H} 1 \mathrm{i}$} & High & 0.2 & 47.6 & 0.0526 \\
\hline & Low & 4.0 & 49.4 & 1.050 \\
\hline \multirow[t]{2}{*}{ H1o } & High & 0.2 & 46.5 & 0.0523 \\
\hline & Low & 23.0 & 30.0 & 1.054 \\
\hline \multirow[t]{2}{*}{$\mathrm{H} 2$} & High & 0.2 & 49.5 & 0.0521 \\
\hline & Low & 4.0 & 50.8 & 1.061 \\
\hline \multirow[t]{2}{*}{$\mathrm{H} 3$} & High & 0.33 & 49.3 & 0.105 \\
\hline & Low & 9.0 & 50.5 & 2.107 \\
\hline \multirow[t]{2}{*}{$\mathrm{H} 4$} & High & 0.33 & 49.0 & 0.105 \\
\hline & Low & 9.0 & 50.1 & 2.097 \\
\hline \multirow[t]{2}{*}{ H5 } & High & 0.36 & 48.8 & 0.104 \\
\hline & Low & 9.0 & 50.0 & 2.097 \\
\hline \multirow[t]{2}{*}{ H6 } & High & 0.2 & 47.9 & 0.0525 \\
\hline & Low & 4.0 & 49.9 & 1.058 \\
\hline
\end{tabular}

perconducting Cyclotron Laboratory at Michigan State University (July 2004) we exposed the two flight HETs to a $160 \mathrm{MeV} / \mathrm{n}$ Ni beam. Ni was not a particularly good choice for HET because Ni saturates at least one PHA channel, but a variable length water target/absorber 
was put in the beam to create lower $Z$ fragments. The lower $Z$ fragments produce smaller energy losses and hence smaller cross-talk signals. With the large signals from the Ni beam, we quickly discovered two different forms of crosstalk. The first of these was cross-talk between $\mathrm{H} 1 \mathrm{i}$ and H1o that is due to stray capacitance between H1i and H1o. Normally, events with pulses in both $\mathrm{H} 1 \mathrm{i}$ and $\mathrm{H} 1 \mathrm{o}$ are rejected as events where the particle trajectory traversed both H1i and H1o. The cross-talk is only about one part in 400, so the key point is to not reject events in which one of two $\mathrm{H} 1$ pulse heights is due to cross-talk.

The second type of cross-talk occurs between channels inside of PHASIC 1. Cross-talk in PHASIC 1 is negligible with regard to obtaining residual energy losses in the stack $(\mathrm{H} 2-$ $\mathrm{H} 5$ or H3-H5). The critical part is to determine whether a signal in H6 is due to cross-talk or not so that stopping particles with large energy losses in the stack don't get erroneously put in the penetrating particle queue. If the H6 pulse height is small, one must unpack the raw pulse heights and gain bits of $\mathrm{H} 2-\mathrm{H} 5$ in order to determine whether the $\mathrm{H} 6$ pulse height is real or is just due to cross-talk. It is to be emphasized that the on-board software identifies both types of cross-talk and eliminates their effects.

\subsubsection{MISC and Front-End Logic}

The MISC, HET front-end logic, and UARTS for communicating with SEP Central are contained in the Actel FPGA. The HET design is similar to the LET design, but the implementation was different. In particular, the layout at Caltech was performed by manual graphical schematic entry. The HET design was implemented using Verilog (a high-level language for describing electronic circuits) and computer-automated layout. The HET MISC has been named the CPU24, to distinguish it from the Caltech MISC (named the P24).

CPU24 is a 24-bit processor with a dual-stack architecture chosen to be optimal for using the Forth kernel (see Mewaldt et al. 2007b). For HET, programming was done using the 32 native MISC instructions rather than Forth. The processor design is sufficiently simple to allow implementation within an Actel RT54SX72S FPGA.

CPU24 employs a reduced instruction set with four 6-bit instructions packed into 24-bit words. The most significant bit (MSb) of each instruction designates an I/O bus operation when set. For I/O bus instructions (the I/O bus will be referred to as the $\mathrm{G}$ bus) the second MSb specifies a write when set and a read when cleared. The remaining 4 bits specify one of $16 \mathrm{G}$ bus addresses. For non-G-bus instructions, the MSb is cleared and the remaining 5 bits specify one of 32 possible instructions.

Following is a list of CPU24 features:

- 24-bit address and data buses

- 6-bit RISC-like CPU instructions

- 4-deep instruction cache

- 17-deep data stack

- 33-deep return stack

- 11 prioritized interrupts

- stack underflow/overflow detection

- clock gating, ability to turn off processor clock during idle time

- stack register clocks individually generated for power saving

- Boot ROM, executes on power up or reset, loads program via UART

- Instruction rate of 6.4 million instructions per second

- Instruction types:

$\circ$ arithmetic and logical

$\circ$ memory access 
$\circ$ register and stack

- program control

$\circ$ input/output.

Details of CPU24 are specified in "CPU24 Microprocessor User's Manual for STEREO HET" (Baker 2003). There are several functional differences between the CPU24 and the P24. The most important of these is stack underflow/overflow detection in the CPU24. This was found to be very useful for finding bugs in the flight software.

The Actel also contains two UARTs. The first is for receiving commands and sending command echoes from/to SEP Central. The second UART (transmit only) is for sending science data to SEP Central. The UARTs all run at $57.6 \mathrm{~K}$ baud.

The Actel also contains

- PHASIC read/write control registers

- a counter that counts valid coincidence pulses

- a front-end livetime counter

- ADC and DAC input/output control signals

- Two extra general purpose input/output registers.

The HET pulse-height controller flow is illustrated in Fig. 16. This figure shows, among other things, how the front-end livetime counter is implemented. This counter measures the time that the PHASICs are enabled to accept a new event (5 $\mu$ s ticks).

MISC tasks will be enumerated and discussed in Sect. 3.

\subsection{Geometry Factors}

\subsubsection{Stopping Particles}

The geometry factors are essentially independent of kinetic energy and particle type. The geometry factor for particles that pass through $\mathrm{H} 1 \mathrm{o}$ or $\mathrm{H} 1 \mathrm{i}$ into detector $\mathrm{H} 2$ and don't enter H6 is $0.61 \mathrm{~cm}^{2}$-steradian. The geometry factor for particles that pass through H1i into $\mathrm{H} 2$ and don't enter $\mathrm{H} 6$ is $0.10 \mathrm{~cm}^{2}$-steradian. Note that during high-rates mode (described in Sect. 3.6.1), particles with $Z>2$ that pass through either H1i or H1o are accepted for analysis, whereas particles with $Z \leq 2$ are only accepted if they pass through H1i.

\subsubsection{Penetrating Particles}

The geometry factor for penetrating particles that pass through $\mathrm{H} 1 \mathrm{i}+\mathrm{H} 1 \mathrm{o}$ and exit through H6 is $0.61 \mathrm{~cm}^{2}$-steradian. The geometry factor for penetrating particles that pass through $\mathrm{H} 1 \mathrm{i}$ and exit through $\mathrm{H} 6$ is $0.10 \mathrm{~cm}^{2}$-steradian. Similar to the stopping particles, penetrating particles with $Z \leq 2$ in high-rates mode are only accepted if they pass through H1i. Figure 17 shows a simulation of forwards and backwards protons and He between 40 and $100 \mathrm{MeV} / \mathrm{n}$. This figure shows that the forwards and backwards particles are essentially separated below $100 \mathrm{MeV} / \mathrm{n}$. Above $100 \mathrm{MeV} / \mathrm{n}$ the tracks rapidly merge.

\section{Onboard Software}

\subsection{Introduction}

The onboard software performs the following tasks: 
- supports the PHASICs (establishes their logic state; reads pulse heights and singles rates and transfers them to the MISC; controls logic terms that define valid coincidences; and controls the on-board pulsers)

- processes pulse height events to bin them into predefined particle and energy bins

- processes all commands

- controls housekeeping measurements such as detector leakage currents and temperatures, and

- formats data into telemetry packets at the end of each major frame and transmits them to SEP Central.

Fig. 16 Illustrates the controller which controls the processing of pulse height events. It also shows how the front-end livetime counter is implemented

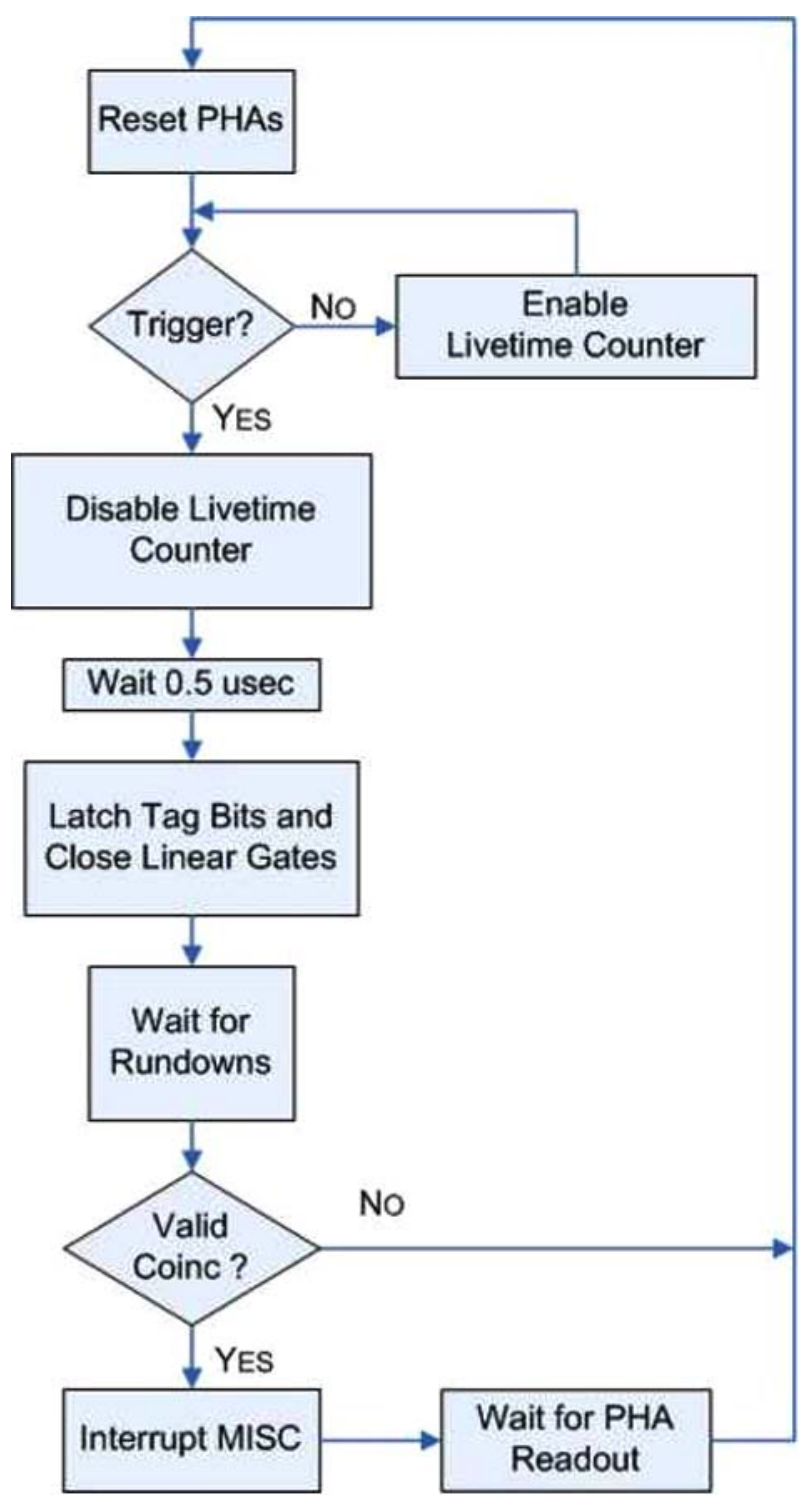


Fig. 17 Simulation of forwards (light blue) and backwards (dark blue) protons and He between 40 and $100 \mathrm{MeV} /$ nucleon. This figure shows that the forwards and backwards particles are essentially separated below 100 $\mathrm{MeV} /$ nucleon. Above 100 $\mathrm{MeV} /$ nucleon (not shown) the tracks rapidly merge

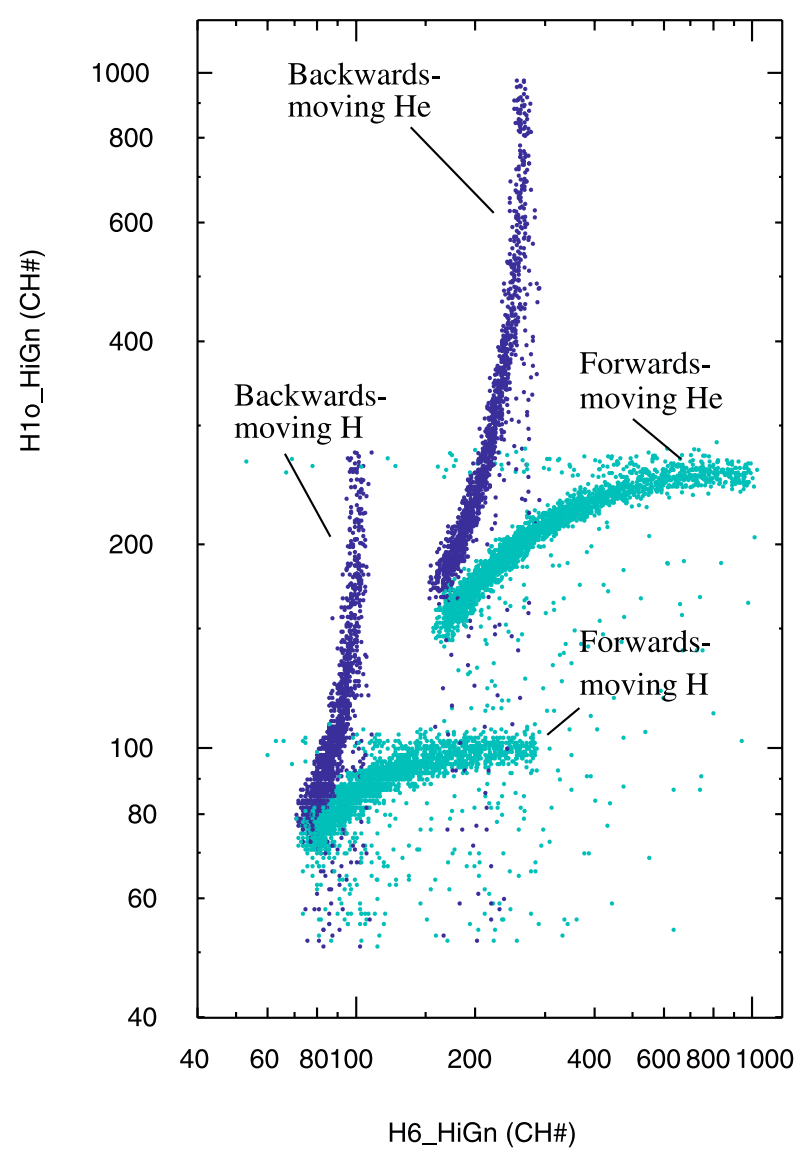

\subsection{Flight Software Architecture}

The HET flight software operates in a background loop with interrupt service routines to respond to external events. On power-up, a one-time bootup section executes before interrupts are enabled and the background loop begins executing. The bootup code loads the remaining on-board code and all the on-board tables from EEPROM in SEP Central, sets up communication buffers, initializes the PHASICs and other hardware, and then enables interrupts and transfers control to the background routine.

The HET software uses the first 8 of 11 prioritized interrupts supported by the CPU24 design. Table 5 lists the interrupt assignments.

Interrupts 0,1 , and 2 signal that the corresponding communications tasks are complete. Interrupt 6 is used during code development but is disabled during flight.

Major frames start on one-minute boundaries. HET telemetry packets are transmitted to SEP Central at assigned seconds so as not to conflict with other subsystems that also transmit telemetry to SEP Central.

\subsection{Flight Software Development Environment}

The decision was made to program the MISC using its native commands rather than Forth, the language used by the LET and SEP Central MISCs. For this a MISC compiler was 
Table 5 CPU24 interrupts

\begin{tabular}{ll}
\hline int 0 & UART \#1 (command) receiver data ready \\
int 1 & UART \#1 (command) transmitter buffer empty \\
int 2 & UART \#2 (telemetry) transmitter buffer empty \\
int 3 & Event interrupt \\
int 4 & One second interrupt \\
int 5 & One minute interrupt \\
int 6 & Stack problem \\
int 7 & Timer \\
\hline
\end{tabular}

Table 6 Compressed event header (16 bits)

\begin{tabular}{lllll}
\hline $15-13$ & 12 & 11 & $10-3$ & $2-0$ \\
\hline Category & High Rate Mode bit & Stim bit & SWCtr \# & \# of PHs in the event \\
\hline
\end{tabular}

written. In addition, a MISC emulator was created in software that allows the programmer to step through the on-board code and, for example, be able to examine the stack contents at any step. In addition, connection of the instrument via ground support electronics to the Internet was enabled early on. In this way it didn't matter whether the instrument was sitting on a bench next to us, was at Caltech on the other side of the country, or was in integration and test at some remote location.

\subsection{Onboard Particle Processing}

To transmit the pulse heights of a single HET particle event to the ground can require up to 16 bytes whereas a count of tens of thousands of events can be transmitted using only two bytes. Due to the limited telemetry allocation for HET it is not possible to send a large number of pulse height events to the ground. For this reason it is important to analyze individual particle events on board and bin the particles into software counters that count the numbers of particles of each type in different kinetic energy intervals. These different software counters for HET are listed in the Appendix. Note that some of these counters are counting background events and events from the on-board pulser. The latter are intended to determine live times and will be discussed further later. The primary purpose of transmitting pulse height events to the ground is to verify that the on-board analysis is being done correctly. In addition, particle types without software counters can be studied to some degree.

The on-board algorithm for stopping particles uses the classic $\mathrm{d} E / \mathrm{d} x \times E^{\prime}$ method of particle identification. Penetrating particles are also accepted and analyzed by a separate algorithm in order to extend the energy range. The expected locations of particle tracks were calculated by a Monte Carlo program that uses energy loss algorithms based on scaling proton range-energy formulae given by Andersen and Ziegler (1977).

HET pulse height events in telemetry are compressed from 3 bytes to only 2 bytes per pulse height and include an event header. The event header has the format as shown in Table 6.

The categories referred to in Table 6 are presented in Table 7.

The category values correspond to different readout queues (see also Figs. 18a, 18b). Note that the coincidence logic can be set to accept $\mathrm{H} 1$ only events as well as events that require an $\mathrm{H} 1$ and $\mathrm{H} 2$ coincidence. To date, $\mathrm{H} 1$ only events have not been accepted on-board. 


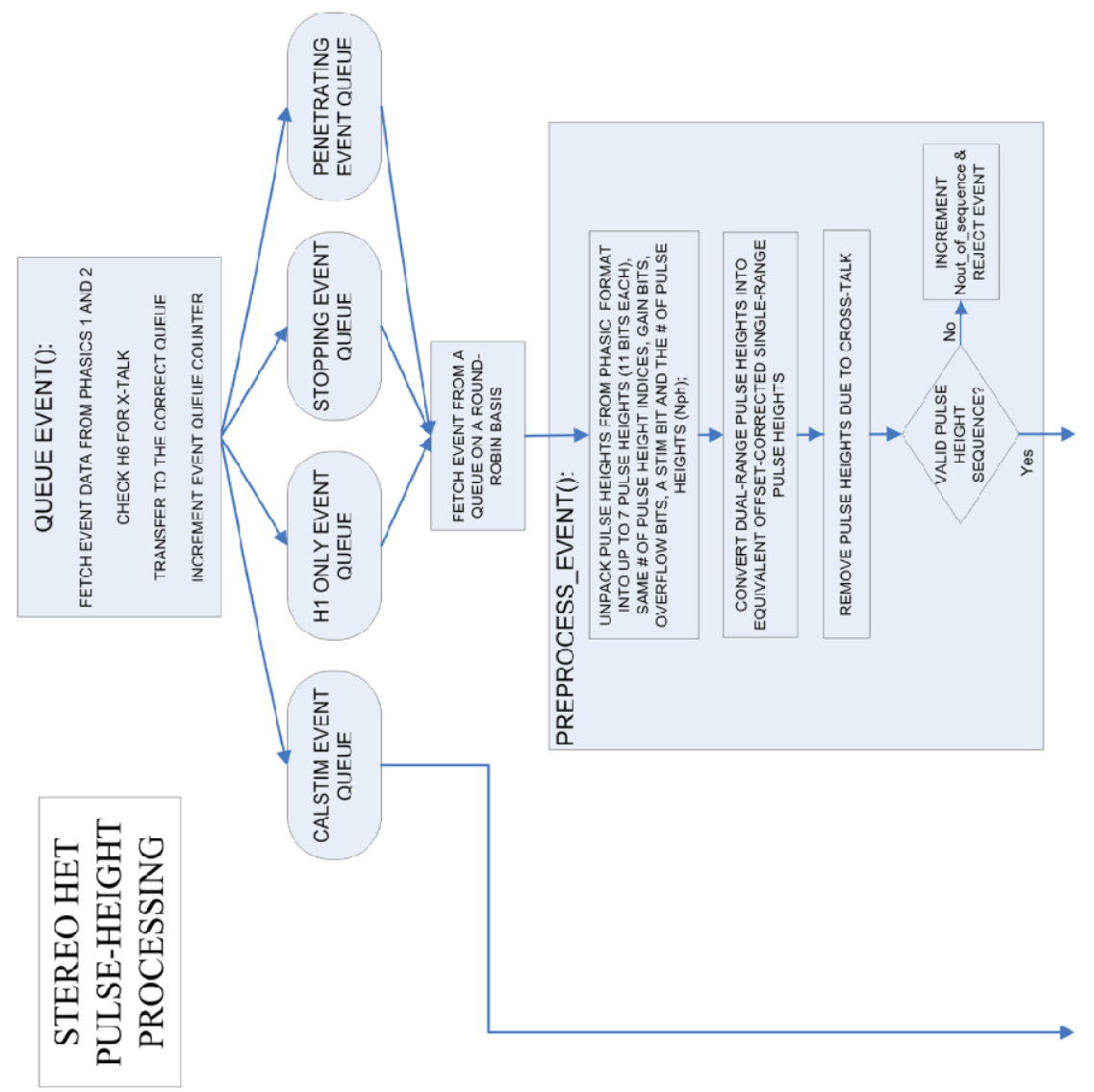

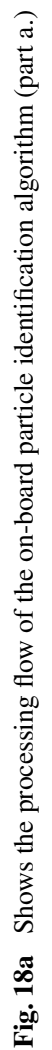




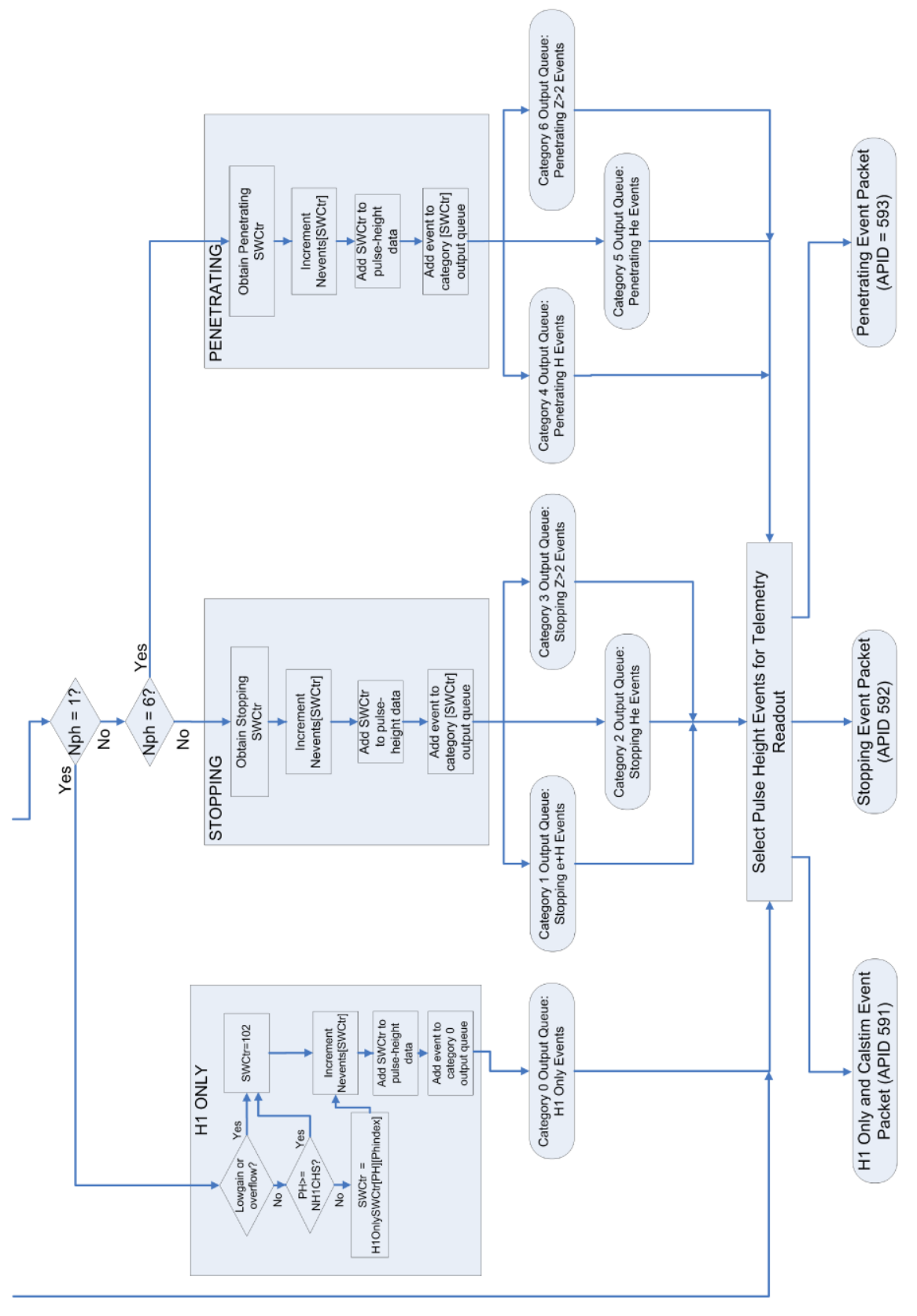

O) 
Table 7 Event Categories

\begin{tabular}{ll}
\hline Category & Description \\
\hline 0 & H1 only \\
1 & Stopping protons and electrons \\
2 & Stopping He \\
3 & Stopping particles with $Z>2$ \\
4 & Penetrating protons \\
5 & Penetrating He \\
6 & Penetrating particles with $Z>2$ \\
\hline
\end{tabular}

Table 8 Compressed event PH format (16 bits)

\begin{tabular}{llll}
\hline $15-13$ & 12 & 11 & $10-0$ \\
\hline PHA index & High gain bit & Overflow bit & Pulse height \\
\hline
\end{tabular}

The compressed format of individual pulse heights is presented in Table 8, where PHA Index has the values: 0 for $\mathrm{H} 1 \mathrm{i}, 1$ for $\mathrm{H} 1 \mathrm{o}, 2$ for $\mathrm{H} 2,3$ for $\mathrm{H} 3,4$ for $\mathrm{H} 4,5$ for $\mathrm{H} 5,6$ for $\mathrm{H} 6$, and 7 for Invalid, and the High Gain bit is 0 for Low Gain.

Note that one can transform the compressed event data back into the raw data format as well as obtain additional information for each event (category, high-rates mode bit, stim bit, and the software counter number assigned on board).

The overall on-board processing flow is illustrated in Figs. 18a and 18b. When the frontend logic issues a valid coincidence interrupt, the MISC reads the event from the PHASICs and the process queue_event() is initiated. queue_event's task is to queue the incoming event into one of 4 different input queues (FIFOs) as well as capture the value of the stim bit and the number of pulse heights in the event.

Calstim events are calibration events that are generated at a low rate $(<1 /$ second $)$ by triggering all 7 on-board pulsers simultaneously. For these events, the pulser DACs are increased by one step per minute during minutes $0-8$ out of every 15 minutes. The resultant events are used to verify the linearity and overall performance of the PHASICs. These are placed in the Calstim event queue (256 3-byte words deep).

If the event is not a Calstim event, then the pulse height index values are determined from the Chip Address and PHA Address values and examined. If there is an H6 pulse height it is checked for cross-talk and the event is correspondingly queued as a stopping event or as a penetrating event. Each queue slot holds the number of pulse heights in the event and the event itself in raw format. The $\mathrm{H} 1$ only queue, the stopping queue and the penetrating event queue can each hold 256 events. The Calstim event queue can hold up to 32 events.

The next step in the event processing is to fetch an event from one of the input queues (other than the Calstim event queue) on a round-robin basis. The event is then handed to the routine preprocess_event(), which performs the sequence illustrated in Fig. 18a. The event will be rejected if the pulse heights are not in $\mathrm{H} 1$ (either $\mathrm{H} 1 \mathrm{i}, \mathrm{H} 1 \mathrm{o}$, or possibly H1i followed by H1o), H2, H3, H4, H5, H6 order. This sequence check is intended to eliminate events for which there are more than two incident particles or possibly events with cross-talk. If accepted, the processing continues as shown in Fig. 18b. The task of the blocks marked H1 only, Stopping, and Penetrating is to determine the proper software counter number, increment this counter by one, and add the event to one of 7 different output queues together with the software counter number. This is also shown in Fig. 18b. The queues for categories 3-6 can each hold up to 64 events. The category 1 and 2 queues can each hold up to 128 
Fig. 19a A 2-D histogram of simulated stopping particles is shown overlaid on top of the stopping particle response table. The response table is plotted with a different color for each stopping particle type

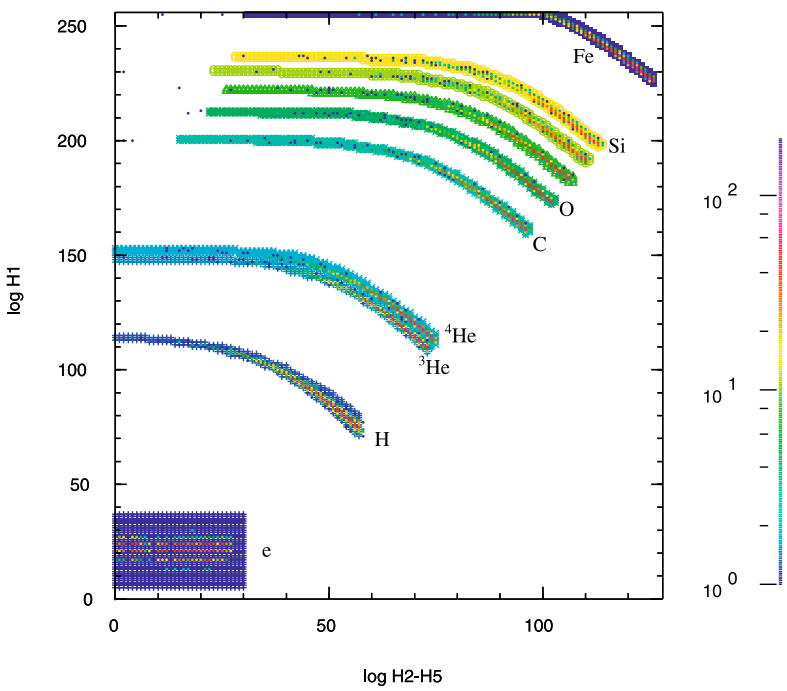

events. At the end of the major frame, events will be selected from these queues on a roundrobin basis and read out into telemetry.

Determination of the stopping software counter number is as follows. Up to 4 logarithmic indices are obtained from the pulse heights by table lookup: $\log \mathrm{H} 1(0-255), \log \mathrm{H} 2(0-255)$, $\log \mathrm{H} 2 \_\mathrm{H} 5$ (0-127), and $\log \mathrm{H} 3 \_\mathrm{H} 5$ (0-127). Here $\log \mathrm{H} 2 \_\mathrm{H} 5$ denotes the index for the logarithm of the sum of offset- and gain-corrected pulse heights $\mathrm{H} 2$ through $\mathrm{H} 5$. There are two corresponding response tables with dimensions $256 \times 128$. The first is for the software counter $(\mathrm{SWCtr}=$ stopSWCtr[ $\left.\log \mathrm{H} 1]\left[\log \mathrm{H}_{2} \_\mathrm{H} 5\right]\right)$ and the second is for the stopping particle type (stopParticleType[logH1] [logH2_H5]). The table size is definitely sufficient for identifying particle types. Quantization of the residual $E$ due to $\log$ conversion (logH2_H5) means that the energy intervals for each particle type are not quite as well matched as one might like. We are considering altering the on-board software to do this binning in linear space to improve this part of the HET performance.

If the software counter does not correspond to a particle track it is assigned a background counter number ( 0 for the category 1 output queue, i.e. for electrons and protons; 1 for the category 2 output queue, i.e. for He; and 2 for the category 3 output queue, i.e. for $Z>2)$ or a livetime stim pulser event counter number $(103,104$, or 105). Otherwise, if there is an $\mathrm{H} 3$ pulse height then a consistency check can be made to see if stopParticleType[logH1][logH2_H5] is the same as stopParticleType[logH2][logH3_H5]. If not, the event software counter is assigned the appropriate background counter. This amounts to the $\mathrm{d} E / \mathrm{d} x$ vs $E^{\prime}$ method being applied first with $\mathrm{H} 1$ as the $\mathrm{d} E / \mathrm{d} x$ detector and then with $\mathrm{H} 2$ as the $\mathrm{d} E / \mathrm{d} x$ detector. The same table can be used for each since the $\Delta x$ detector is $1 \mathrm{~mm}$ thick $\mathrm{Si}$ in each case and because the gains have been matched.

The stopping response tables are illustrated in Figs. 19a and 19b. Figure 19a shows a 2-D histogram derived from simulated particle events overlaid on top of a plot of the stopping particle response table stopPrtclType[logH1][logH2_H5]. Figure 19b shows a sample histogram of stopping particles from the SEP event of 13 December 2006 (see Sect. 5).

Analysis of penetrating events is similar. This multi-dimensional problem has been reduced to two 2-dimensional response planes: the first of these is the $\log \mathrm{H} 1$ versus $\log \mathrm{H} 6$ plane (128 by 128 logarithmic channels for protons and $\mathrm{He}$ ), and the second is the $\log \mathrm{H} 2$ versus logH3_H5 plane (also 128 by 128 logarithmic channels). No accelerator tests were 
Fig. 19b Shows a 2-D histogram of stopping particles in the $\log \mathrm{H} 1$ versus $\log \mathrm{H} 2 \_\mathrm{H} 5$ plane observed during the event of 2006 December 13

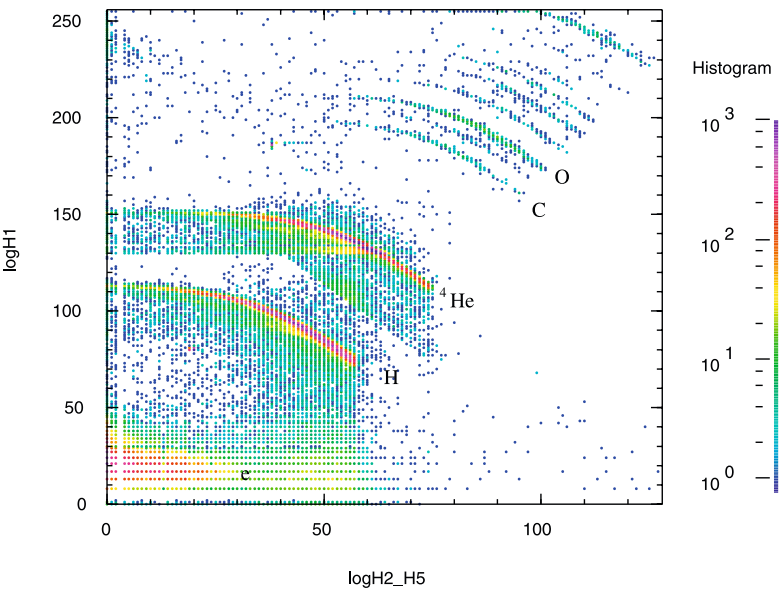

performed with penetrating protons and He, so the current tables are solely based upon the simulated response. An additional complication is that, due to the relatively shallow stack, the forward and backwards tracks merge at a fairly low energy. It is intended to refine the response tables using solar event data.

\subsection{Command Processing}

The default command state, on-board tables, and software for HET are all contained in EEPROM in SEP Central and are loaded into HET at boot up. Changes to the EEPROM contents can be made by transmitting a complete HET EEPROM image to the spacecraft. This takes approximately 10 minutes of commanding. Alternatively, a paging scheme is available that uploads only pages that have changed. The remainder of this section addresses commands that may be used to make changes in HET RAM and in the PHASICs. These changes will of course be lost if HET is turned OFF or is rebooted. Command bits in the PHASICs are refreshed once per major frame by the HET software. The reason for this is that the RAM chips used by the MISC are much more resistant to single-event upsets (SEUs) than the PHASICs.

The command interface between HET and the spacecraft is managed by SEP Central. HET commands transmitted from the ground as CCSDS telecommand packets (CCSDS 2000; see also STEREO MOC to POC and to SSC ICD 2002) are received by the IMPACT IDPU, which in turn forwards them to SEP Central. SEP Central unpacks the commands and routes them to the HET sensor via a bi-directional serial command interface. Command echoes from the HET sensor are routed back to SEP Central and then to the spacecraft via the same path.

Commands can be received by the HET sensor at any time during a major frame. There are two modes for command execution by the HET software: immediate and non-immediate. In immediate execution mode, commands are executed immediately instead of waiting for a major frame boundary. In non-immediate mode, the commands are executed at the major frame boundary. Non-immediate mode is the default command execution configuration used for the HET software. The non-immediate mode strategy preserves the integrity of the data for each major frame (i.e. the command state is the same throughout the major frame).

A command that is sent to SEP Central for execution by the HET sensor is preceded by an identifier that informs SEP Central to forward the command to HET, and indicates whether 
the command is ASCII or binary (see below). These command keywords "HET-CMD" and "HET-BIN" are used to identify the HET sensor and precede any sequence of commands routed through SEP Central. These keywords are interpreted by SEP Central and not passed through to the HET sensor.

The HET response for each command is essentially an ASCII command echo. If the keyword in the command received by the HET sensor is not recognized by the software, the response will contain the keyword echo followed by a question mark. The absence of a question mark in the command response signifies confirmation that the command was received and is queued for execution. Immediate execution is indicated in the command echo by an asterisk. SEP Central collects the HET command responses and multiplexes them into packets and transmits them as part of its telemetry stream.

ASCII commands are used to set parameters in the software and to configure the HET front-end electronics and the PHASICs. Different commandable functions for the PHASICs were cited in Sect. 2.3.2. Binary commands are used in conjunction with ASCII commands to load large particle look up tables into the HET MISC RAM.

Binary commands are used to specify the starting memory location in RAM and the number of words to load, followed by the data. The HET software accepts the binary data as a byte stream, buffering it in memory with no interpretation. Once the binary data have been transmitted, a subsequent ASCII command is necessary to interpret and activate the loading of the data. A subsequent ASCII command causes the HET software to copy the buffered byte stream, according to the specified decompression scheme, to a specified memory address.

If the computed checksum matches the received checksum, the $\mathrm{OK}$ echo is sent in the command response. The command response consists of the ASCII keyword "binary" followed by the relative address "A:" where the binary command load was placed in memory, and the number of bytes "N:" in the command load. Additionally, if an error occurred, "ckerr" is present followed by the received and computed checksums.

\subsection{High-Rate Strategy}

\subsubsection{High-Rate Mode}

Historically speaking, particle telescopes have frequently saturated during the largest solar particle events. In order to increase HET's capability to make measurements during intense events, the front detector on HET has been segmented into a small central detector (H1 inner, denoted as $\mathrm{H} 1 \mathrm{i})$ surrounded by a circular ring ( $\mathrm{H} 1$ outer, denoted by $\mathrm{H} 1 \mathrm{o}$ ). The normal mode is the low-rates mode, where both $\mathrm{H} 1 \mathrm{i}$ and $\mathrm{H} 1 \mathrm{o}$ are fully enabled. In this mode, electrons, protons and $\mathrm{He}$ are detected and processed by either H1i or H1o through their high-gain PHA channels. When the H1i singles rate exceeds a (commandable) level, the MISC automatically switches the instrument to high-rates mode. This switching occurs at a major frame boundary and involves disabling the H1o high-gain PHA channel. Higher $Z$ particles that pass through the H1o detector continue to trigger the H1o low-gain PHA channel. After launch we discovered that low energy stopping He, which normally is processed by the H1o high-gain PHA channel, will be accepted and processed by the low gain channel when the high-gain channel is disabled. High energy stopping He, however, is unable to trigger the low-gain channel. This complicates the He high-rates analysis, because He now has two different geometry factors, depending on the He energy. We could raise the H1o low-gain threshold and eliminate this effect, but so far we have elected to not do so. When the H1i singles rate falls below a certain (commandable) count rate, normally half the rate that causes 
HET to go into high-rate mode, the MISC re-enables the low-rate mode. To date the H1i rate at which the high-rate mode begins has been set at 400 counts per second, a rather low value.

There are some draw-backs to the high-rate mode, but we don't consider them serious. One is that in low-rates mode, the $\mathrm{H} 1 \mathrm{i}$ and $\mathrm{H} 1$ o detectors act as guards for particles that pass through both detectors. In high-rate mode, the H1o threshold is raised too high to detect low $Z$ particles that intersect both $\mathrm{H} 1 \mathrm{i}$ and $\mathrm{H} 1 \mathrm{o}$. In particular, ${ }^{4} \mathrm{He}$ that passes through the H1i edge can fall down into the ${ }^{3} \mathrm{He}$ region of the H1 versus H2_H5 SWCtr matrix. On the other hand, the stopping-particle consistency check described in Sect. 3.4 can help to eliminate these ${ }^{4} \mathrm{He}$ from being misidentified as ${ }^{3} \mathrm{He}$. One reason that this is not really a serious problem is that ${ }^{3} \mathrm{He}$-rich events are generally small events, ones that are too small to trigger the high-rate mode.

\subsubsection{Livetimes}

The front-end livetime is measured by counting pulses from a clock running at $2 \times 10^{5}$ pulses per second in a counter that is gated off when the MISC is not available to accept new events (see Fig. 16).

It was mentioned in Sect. 3.4 that Calstim events are generated during minutes $0-8$ out of every 15 minutes. During minutes $9-14$, the pulsers are set to generate events that correspond in turn to each of the different event categories 0-5 (see Sect. 3.4) at a rate of 600 per minute. These events are processed on-board as if they were due to particles. They are counted in software counter numbers 103-108. These counters are located in low-background regions of their respective categories. The number that get counted in a given minute is a measure of the livetime for on-board processing events in the corresponding category. This overall sequence of pulsing (minutes 0-14) can be disabled by command. These events have been chosen so that their counting rate is unaffected by the high-rate mode. Category 6 does not have a livetime counter.

The livetime counters measure the total livetimes for categories 0-5. These livetimes are in general shorter than the front-end livetime because, at high rates, the MISC may not be able to obtain the software counter number for every pulse height event that it receives. Figure 20 shows the front-end livetime and the 3 stopping event category livetimes during the large event of 13 December 2006. It can be seen that the front-end livetime is only slightly longer than the other livetimes, and also that the livetimes were reasonably high, even at the peak of the event.

\subsection{Telemetry Formatting}

\subsubsection{Packet Strategy}

The telemetry output of the STEREO IMPACT HET telescope contains CCSDS packets in 8 different data formats:
A. Rate packets
B. Status and single $\mathrm{PH}$ packets
C. Stopping particle $\mathrm{PH}$ packets
D. Penetrating particle $\mathrm{PH}$ packets
E. Table status dump
F. Beacon packets 
STEREO Ahead HET Livetimes

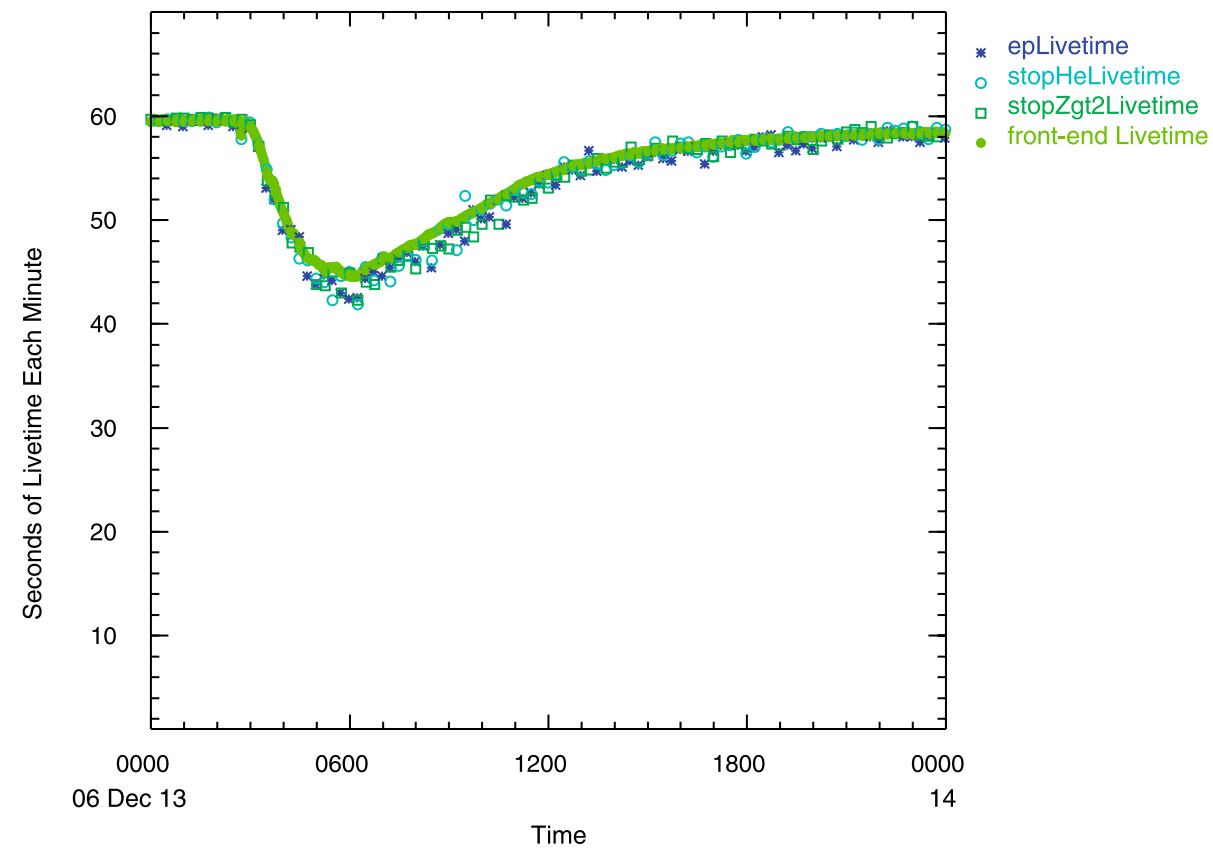

Fig. 20 Plots the front-end livetime and the stim event livetimes versus time during the ground level event which started on December 12, 2006. Note that the livetimes measured by the stim pulser are only slightly below the front-end livetime at the peak of the event (minimum livetime)

\section{G. Housekeeping data packets}

H. Raw event packets (non-flight).

Each CCSDS packet is 272 bytes long and begins with an 11-byte CCSDS header. The quantities of primary interest in the CCSDS header are the ApID, which identifies the specific packet type, and Universal Time in seconds since 1 January 1958 00:00:00. Packet types A.-E. have ApIDs 590-594; Packets F. and G. are sent to SEP Central with ApIDs of 599 and 598, respectively; Packet H has ApID 597.

After individual particle pulse height (PH) events are recorded by HET, the onboard processing algorithm identifies particle species and energies and bins the particles in "software rate counters", as distinguished from hardware counters in the front-end electronics. The identification of these counters is given in the Appendix. In addition to binning all the particles, samples of the raw $\mathrm{PH}$ events are selected in 8 categories (including the Calstim events) for inclusion in the telemetry stream. Note that PH events can vary in length from 2 to 16 bytes (always even).

All rates are log compressed from 24-bit to 16-bit quantities for telemetry (5 bits for the characteristic, and 11 bits for the mantissa with leading 1 suppressed; integers up through 4096 are recovered exactly). Quantities longer than one byte are written into the packets least significant byte first with the exception of the CCSDS header, which is most significant byte first.

In normal operation, HET generates 6 primary packets during a one-minute frame; these might be formatted as follows: one A packet, one $\mathrm{B}$ packet, three $\mathrm{C}$ packets, and one $\mathrm{D}$ 
Table 9 HET Beacon data

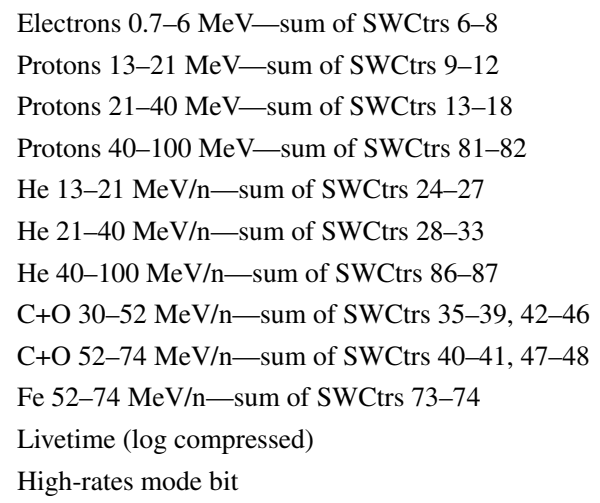

packet. E packets are multiplexed out at a rate that can be selected by command. In flight, typically, E packets replace a $\mathrm{PH}$ packet once every 15 minutes to produce a complete dump every $\sim 7$ days. F and $\mathrm{G}$ packets contribute the HET portions of the SEP Beacon and housekeeping data, respectively, i.e. they are sent to SEP Central, which in turn combines them with data from other IMPACT SEP instruments to create combined Beacon (ApID 624) and housekeeping packets (ApID 577). Command echoes are in packet 576. Sample PHs to fill the stopping particle $\mathrm{PH}$ packets are obtained by a round-robin sampling of the three stopping-event queues (categories 1-3). Similarly, sample PHs to fill the penetrating particle $\mathrm{PH}$ packets are obtained by a round-robin sampling of the three penetrating event queues (categories 4-6).

Each HET packet ends with a checksum as the last byte. The checksum is calculated such that if all 272 bytes of the packet are added together the lowest order byte is hex 00 .

\subsubsection{Beacon Packet}

STEREO broadcasts Beacon data continuously to be used by NOAA and others to monitor and forecast interplanetary space weather (see, e.g., Luhmann et al. 2007). Included are both imaging and in situ data. HET Beacon data are transmitted to SEP Central to be incorporated into a single telemetry packet (ApID 624 decimal) that contains Beacon data for the entire IMPACT SEP suite. SEP Central time tags the data to correspond to the start of the data accumulation time. The HET Beacon data include the quantities presented in Table 9.

\subsubsection{Housekeeping Packet}

HET housekeeping data are transmitted to SEP Central to be incorporated into a single telemetry packet for the entire IMPACT SEP suite. HET housekeeping includes 2 temperatures, subcommutated threshold settings, digitized preamplifier DC output values (the preamplifiers are selected by commands), leakage current data, 2 bytes of command error flags, a checksum for all the tables taken together, the major frame number, the number of invalid triggers, and the software version number.

\section{HET Resources}

The HET uses the following resources: 
Weight: $595 \mathrm{~g}$

Power: $359 \mathrm{~mW}$ average, $498 \mathrm{~mW}$ maximum (excluding power supply inefficiency)

Bit-rate: 218 bits/s.

The HET weight reported here includes the telescope plus the electronics board. It does not include the portion of the SEP Central walls to which HET is mounted (see Fig. 14a). These walls will be referred to as the HET electronics enclosure. The HET telescope is 82 $\mathrm{mm}$ long and has a maximum diameter of $68.5 \mathrm{~mm}$. The overall height, measured from the top of the telescope to the bottom of the electronics enclosure, is $206 \mathrm{~mm}$. The electronics enclosure has maximum dimensions $133 \mathrm{~mm}$ high, $172 \mathrm{~mm}$ wide, and $95 \mathrm{~mm}$ deep.

The bit-rate includes HET's shares of the housekeeping and Beacon packets.

The HET does not require any heaters.

\section{System Level Tests}

\subsection{Bench Tests}

The HET electronics performance was tested at $-20^{\circ} \mathrm{C}, 0^{\circ} \mathrm{C},+20^{\circ} \mathrm{C}$, and $+40^{\circ} \mathrm{C}$. Pulse height thresholds, offsets and analyzer linearity curves were measured using an Ortec 448 Research Pulser. The results are reported in Tables 4a, 4b. Particle response performance was measured using pulsers (including the on-board stim pulsers), a ${ }^{106} \mathrm{Ru}$ electron source, and muon runs.

\subsection{Accelerator Runs}

As mentioned earlier, accelerator runs were made at the National Superconducting Cyclotron Laboratory at Michigan State University in East Lansing. The initial runs were marred by unexpected cross-talk. The flight units were no longer available for our second trip to MSU in May 2006, so we did runs with the engineering test unit. The on-board tables were modified by replacing the $\mathrm{Fe}$ response with the expected Ca response (the test beam was ${ }^{40} \mathrm{Ca}$ at $140 \mathrm{MeV} / \mathrm{n}$ ). Air between $\mathrm{H} 1$ and $\mathrm{H} 2$ was included in the response calculations since the telescope was not placed in a vacuum for these tests. The ETU tests showed that the overall design, including the on-board software, was performing reasonably well. Particle tracks were populated using fragments from the primary beam. Some changes were made to the flight software as a result of the ETU tests. For the purpose of demonstrating the performance of the two flight units, rather than presenting the ETU accelerator data, we will present flight data for the two flight units. In particular, in the next section we present results from a perigee pass and from an SEP event in December 2006.

\subsection{Post-Launch Data}

After launch by a single rocket, the two STEREO spacecraft were placed in elliptical orbits with apogees at the lunar distance. Subsequently the Ahead spacecraft did a lunar swing-by that injected it into its current orbit around the Sun with an aphelion slightly less than 1 AU. The Behind spacecraft performed two lunar swing-bys that injected it into a solar orbit with aphelion slightly greater than $1 \mathrm{AU}$. As a consequence, the Ahead spacecraft will drift ahead of the Earth by about $22.5^{\circ}$ per year, and the Behind spacecraft will drift behind the Earth at the same rate. 


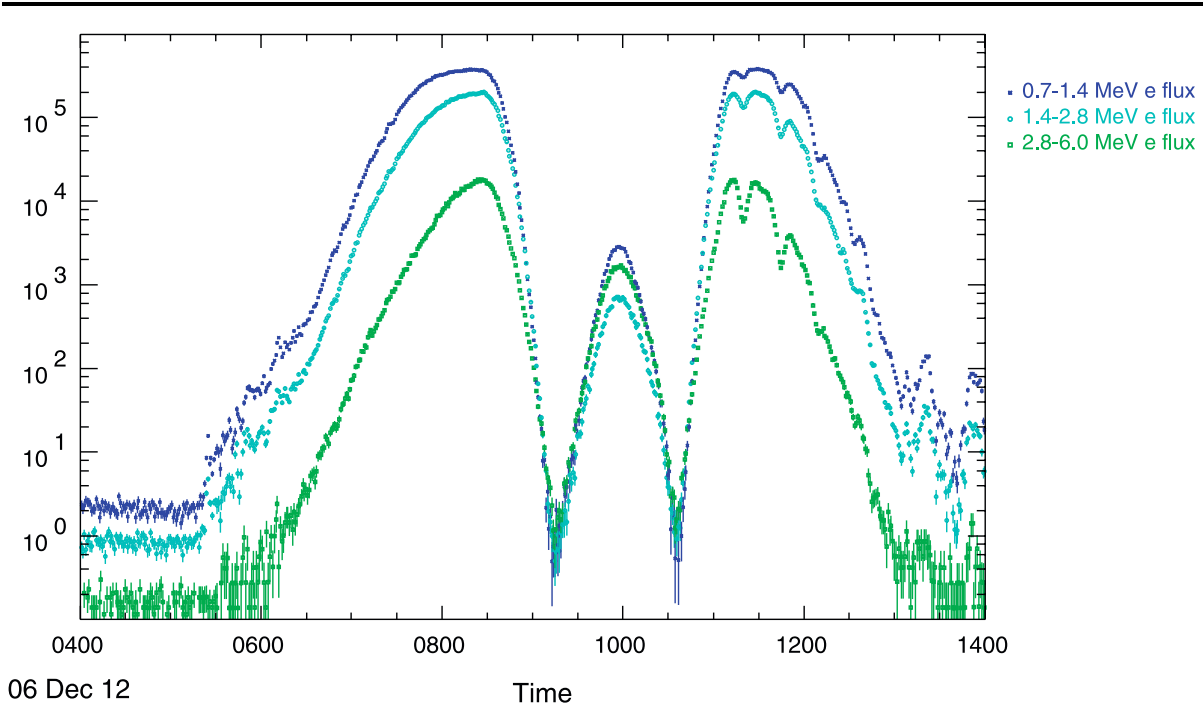

Fig. 21 Shows electron intensities $\left(\# / \mathrm{cm}^{2}-\mathrm{sr}-\mathrm{sec}-\mathrm{MeV}\right)$ for 3 different energy intervals during a perigees pass of the Behind spacecraft

The early post-launch data from both the Ahead and Behind HETs are essentially identical, so in what follows different figures will be chosen for a random spacecraft and will not be shown for both spacecraft.

Figure 21 shows electron intensities in three energy intervals as measured by the Behind spacecraft during the perigee pass on 12 December 2006. The apparent lack of count-rate saturation demonstrates the ability of the HET to perform at high counting rates.

Since launch the Sun has been at the minimum of the 11-year solar activity cycle as measured by sunspot number. Hence it was quite surprising when several large SEP events occurred in December of 2006. The event of 13 December was a Ground Level Event (i.e. it was detected by neutron monitors at sea level). It occurred at a solar longitude of W23. At the peak of this event, HET was processing almost 4000 particles per second. Between 13 December 02:40 and 14 December 14:00, the Ahead HET processed $3 \times 10^{6} \mathrm{H}$ and $2.6 \times 10^{5}$ He.

Figure 22 shows two histograms: the first is for $Z$-values estimated for stopping $\mathrm{H}$ and He pulse height events that were sent to the ground. These estimates were based on the $\mathrm{H} 2$ and H3-H5 energy losses for each event (Stone et al. 1998). Inspection of the first figure shows that the required charge resolution of $<0.2$ charge units is being met for $\mathrm{H}$ and $\mathrm{He}$. The second histogram is for similar estimates of the particle masses. Again, inspection of the figure shows that the desired mass resolution of $<0.2$ mass units is being met for ${ }^{4} \mathrm{He}$.

Figure 23 shows the measured intensity-time profiles for electrons (0.7-1.4 MeV), $\mathrm{H}(20.8-23.8 \mathrm{MeV})$, and $\mathrm{He}(21.1-24.4 \mathrm{MeV} / \mathrm{n})$ for the event of 13 December. Figure 24 shows similar data for two previous solar events from the same active region that produced the event of 13 December. Note how different the time profiles are as compared to those of Fig. 23. One of the objectives of STEREO is to better understand how such different profiles occur. Figure 25 shows a comparison of proton energy spectra measured by ACE/EPAM, SAMPEX, STEREO/IMPACT/LET, and HET. And finally, Fig. 26 shows a 2-D histogram of $\mathrm{H}$ and $\mathrm{He}$ that penetrated the HET telescope, either forwards or backwards. Collectively 

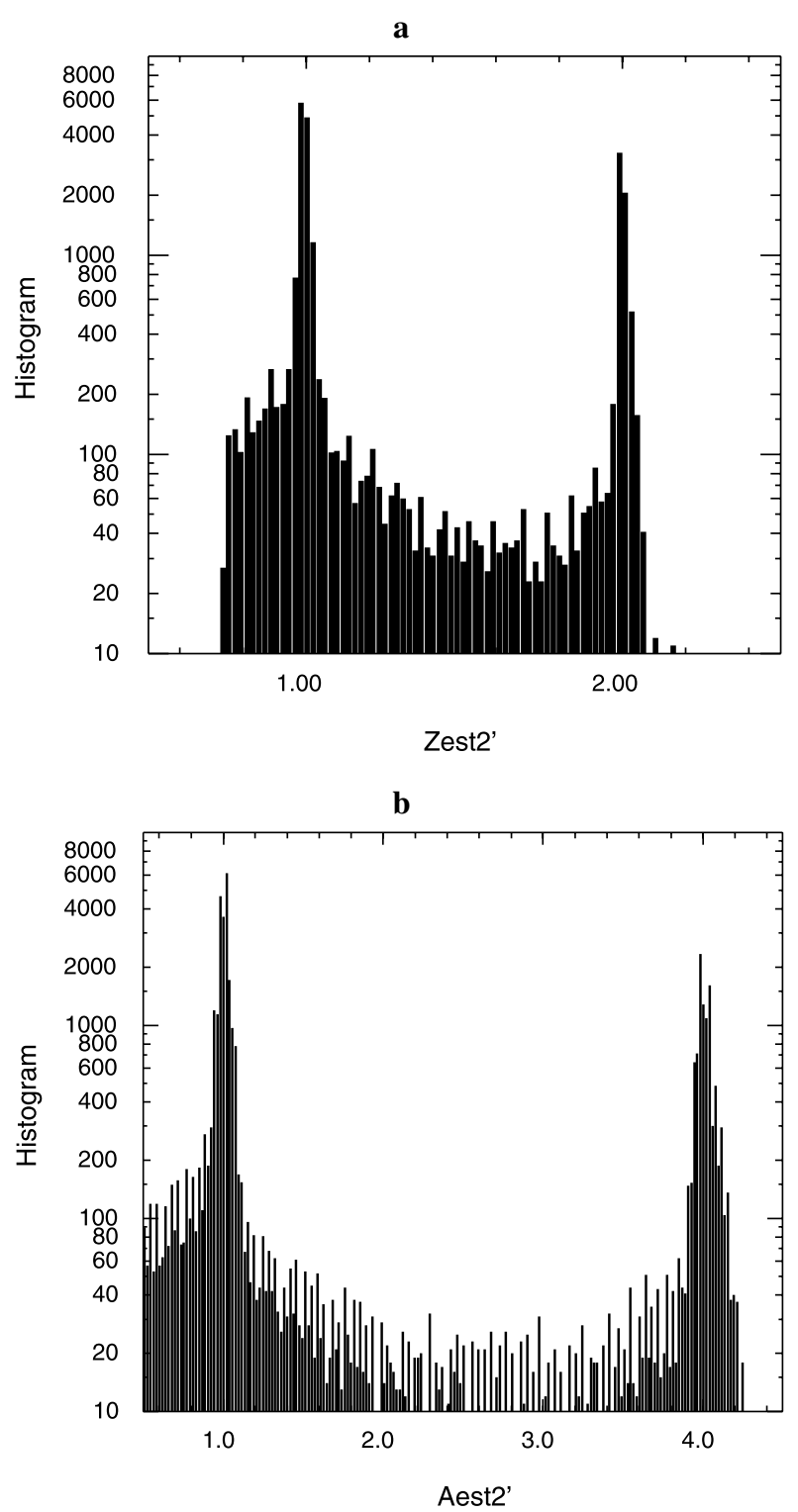

Fig. 22 Histograms of estimated $Z$-values and $A$-values for $\mathrm{H}$ and $\mathrm{He}$ observed during the event of 2006 December $13^{\text {th }}$

these figures illustrate that the HETs are working well, even in solar events that have very high intensities.

Acknowledgements This research was supported by the National Aeronautics and Space Administration at the Goddard Space Flight Center (GSFC). The design, building, and testing of the HETs was only possible with the assistance of a large number of people, whom we wish to acknowledge here. Branislav Kecman at Caltech provided many of the HET electronic parts, in particular the fully tested PHASICs. The many individuals who contributed to the PHASICs are acknowledged in Mewaldt et al. (2007b). Other parts were 
Fig. 23 Shows the measured intensity-time profiles for electrons (0.7-1.4 MeV), $\mathrm{H}$ (20.8-23.8 MeV), and $\mathrm{He}$ (21.1-24.4 MeV/n) for the event of 2006 December 13

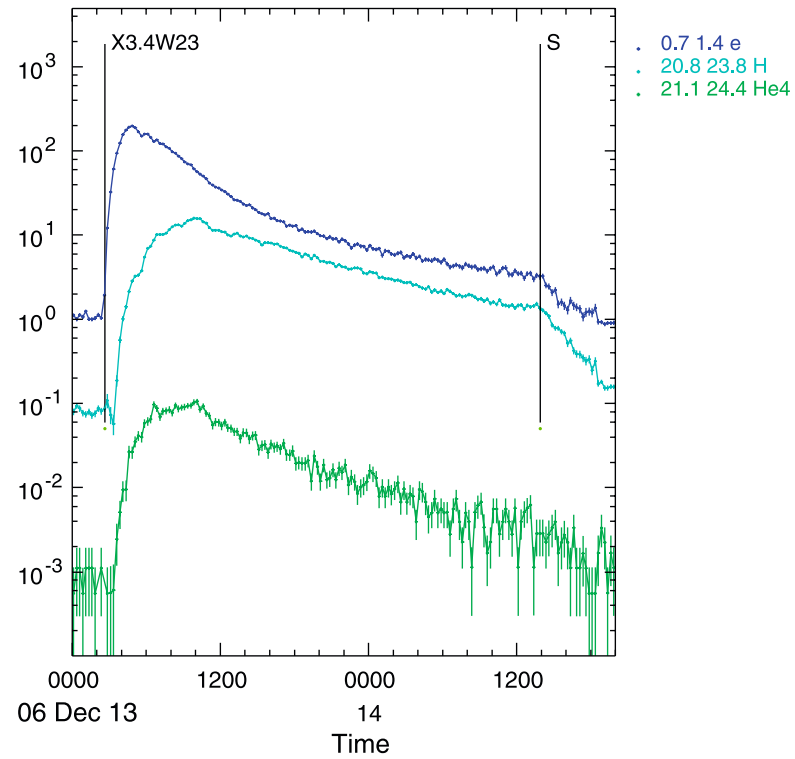

Fig. 24 Shows the

time-intensity profiles of $0.7-1.4$ $\mathrm{MeV}$ electrons, 14.9-17.1 MeV $\mathrm{H}$, and 15.0-17.2 MeV/n He for the time period 2006 Dec 5-11. Note the very different time profiles as compared to the previous figure

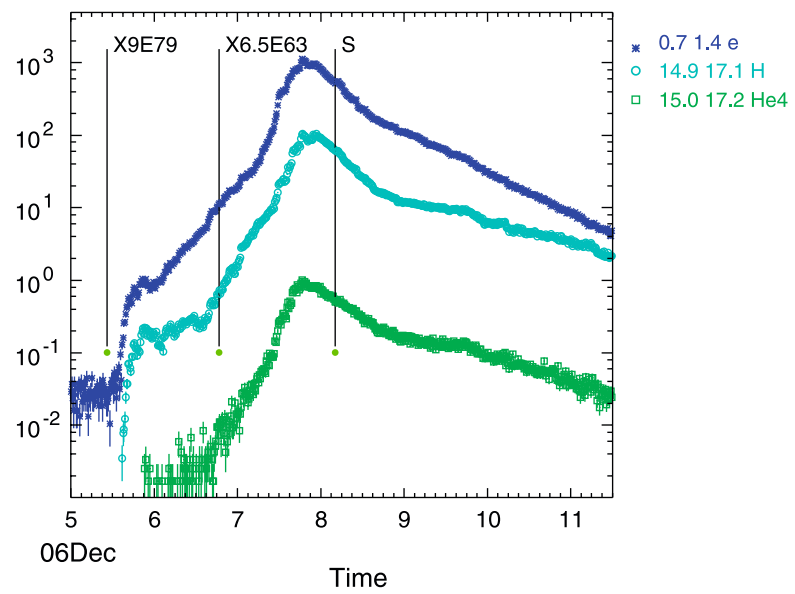

procured by Maxine Windhausen at GSFC. Marci Holzapfel performed the board layout for the HET electronics. Traci Pluchak-Rosnack populated the electronic boards and supervised staking and conformal coating of the boards. Bert Nahory and John Krizmanic performed the detector tests at GSFC. Environmental testing and spacecraft-level testing of the HETs was done together with LET and SEP Central under the guidance of Branislav Kecman, with software support from Bob Radocinski, and other support from personnel at JPL and Caltech. Janet Cunningham was the technician in charge of thermal blanketing of HET/LET/SEP Central. The HET front foils were coated by George Harris. Colin Wilburn of Micron Semiconductor worked with us until significant problems with HET detector leakage currents were resolved. Mechanical modeling of HET/LET/SEP Central was performed by Terry Fan of Swales, Inc. Software support at GSFC was provided by Haydar Teymourlouei.

Accelerator calibrations and performance tests were performed on the HET flight units and subsequently on the HET engineering test unit at the National Superconducting Cyclotron Laboratory (NSCL) at Michigan State University in East Lansing, Michigan. We thank Raman Anantaraman and his support staff at NSCL for their prompt and very professional support. We also thank Sven Geier and Hiromasa Miyasaka at Caltech for their support of the accelerator tests. 
Fig. 25 Shows a comparison of proton intensities measured by ACE/EPAM, SAMPEX, STEREO/IMPACT/LET, and HET. The figure is taken from R.A. Mewaldt et al. (2007a)

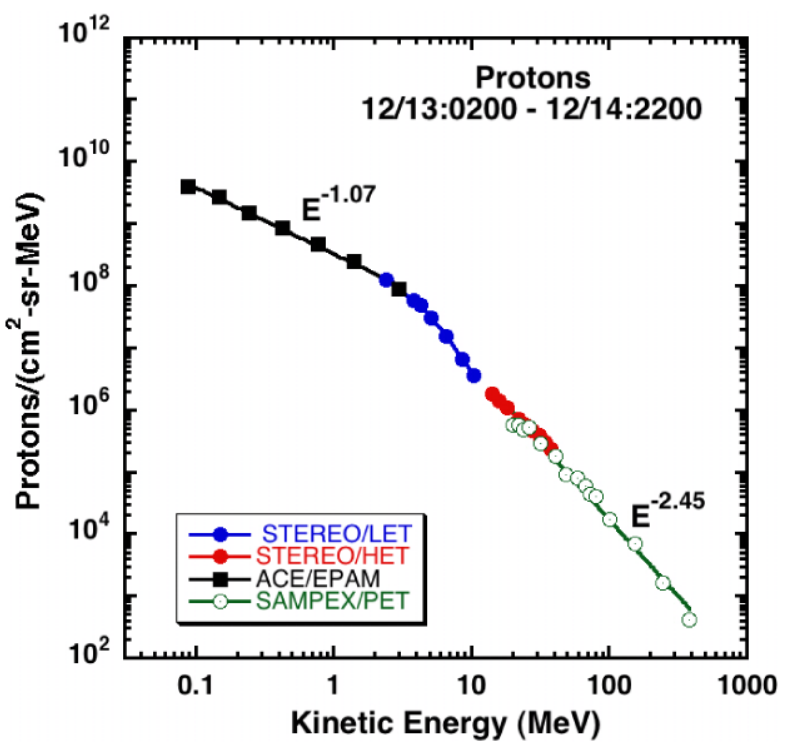

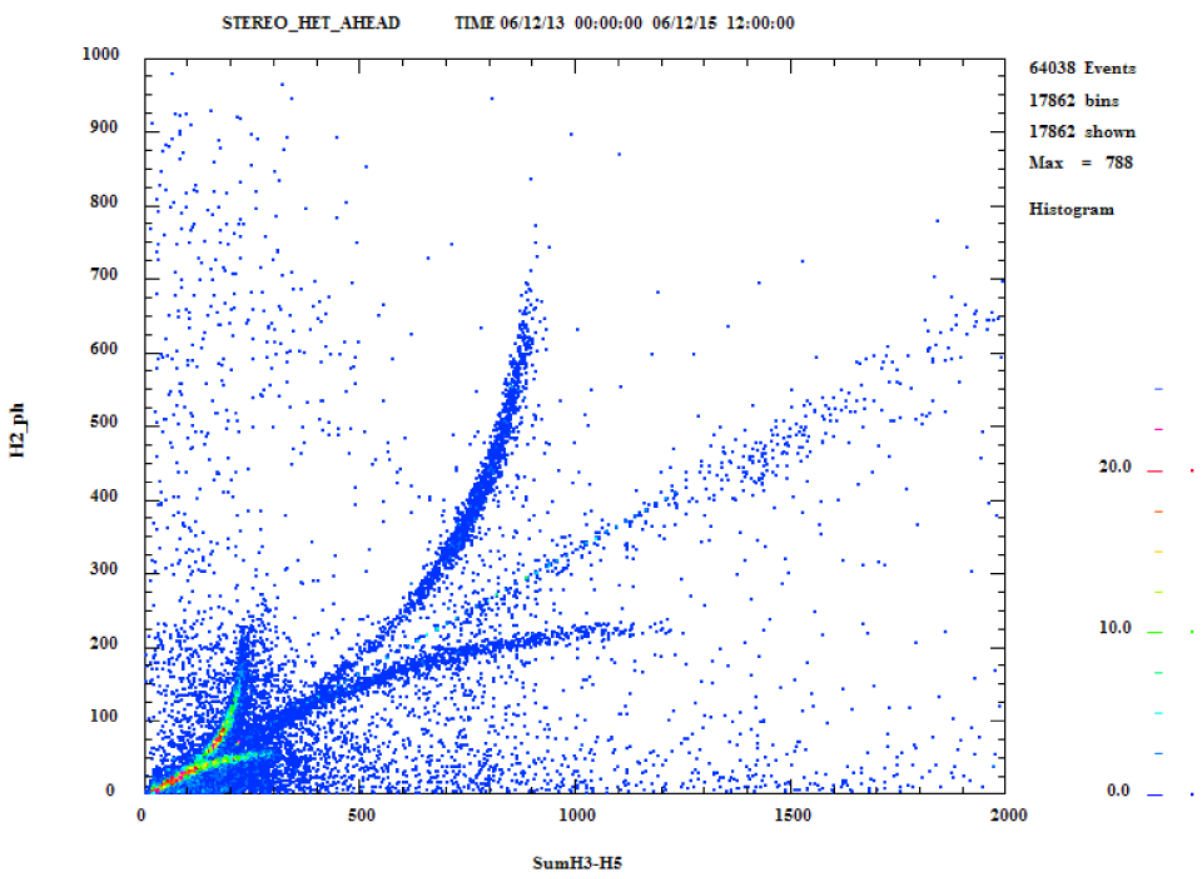

Fig. 26 Shows the HET response for penetrating $\mathrm{H}$ and He. This figure is a 2-D histogram formed from penetrating particle pulse height events transmitted to the ground. The offset-corrected $\mathrm{H} 2$ pulse height is binned and plotted on the $y$-axis and the sum of the offset corrected pulse heights $\mathrm{H} 3-\mathrm{H} 5$ is binned and plotted on the $x$-axis. The number of events in each $x-y$ bin is plotted as a color. The upper tracks are for backwards-moving particles, while the lower two tracks are for forward-moving particles (see Fig. 17) 
We wish to thank the STEREO Mission Project Office at GSFC for their extensive support. Haydee Maldonado was the initial STEREO Project Manager, a role subsequently passed to Nick Chrissotomos. Mike Delmont was the Deputy Project Manager. Joe Davila was the initial STEREO Project Scientist, followed by Mike Kaiser. We thank each of them for their support. We especially wish to acknowledge Lillian Reichenthal, the STEREO Project Instrument Manager for IMPACT, who was a staunch supporter and active member of our team. She prodded us to look at the big picture (schedule, schedule, schedule!) when we were mired in details, and assisted us on a daily basis. Other support from the Project Office was provided by Harry Culver, Therese Errigo, Larry Gibb, Fred Gross, Jerry Hengermihle, Shane Hynes, Mike Jones, Diane Kolos, Tabitha Merchant, Bobby Power, Antonio Reyes, and Steve Wasserzug.

We also wish to thank many individuals at the Johns Hopkins Applied Physics Laboratory (APL), some of them unknown to us, for their support as the spacecraft contractor. In particular, we wish to thank Andy Dreisman, the Deputy Project Manager at APL, and Dave Myers, the primary APL contact for IMPACT. Possible detector contamination by low-level volatile fumes was a constant issue that APL handled effectively.

We wish to thank various individuals at the University of California at Berkeley (UCB). Janet Luhmann is the IMPACT Principal Investigator and we thank her for her active encouragement and support. David Curtis was the IMPACT project manager at UCB. His long professional experience, his easy-going manner under fire, and his ability to keep on top of many different complex issues all at the same time were invaluable to us. Other support has been provided by Peter Berg, Selda Heavner, Ron Jackson, and Peter Schroeder.

Finally, we thank Eric Christian, the STEREO Program Scientist at NASA Headquarters, both for his administrative support and encouragement, but also for his willingness to take night shifts for spacecraftlevel thermal vacuum testing which required 24 hours per day/7 days per week coverage for approximately a month.

\section{Appendix: Nominal HET software counters}

\begin{tabular}{|c|c|c|c|c|c|c|}
\hline $\begin{array}{l}\mathrm{SW} \\
\mathrm{Ctr} \#\end{array}$ & $Z$ & $A$ & $\begin{array}{l}\mathrm{KEmi} \\
(\mathrm{MeV}\end{array}$ & $\begin{array}{l}\text { KEmax } \\
(\mathrm{MeV} / \mathrm{n})\end{array}$ & $\begin{array}{l}\text { Particle } \\
\text { type }\end{array}$ & Cat. \\
\hline 0 & \multicolumn{4}{|c|}{ background for stopping $\mathrm{e}+\mathrm{p}$} & - & 1 \\
\hline 1 & \multicolumn{4}{|c|}{ background for stopping $\mathrm{He}$} & - & 2 \\
\hline 2 & \multicolumn{4}{|c|}{ background for stopping $Z>2$} & - & 3 \\
\hline 3 & \multicolumn{4}{|c|}{ background for penetrating $\mathrm{H}$} & - & 4 \\
\hline 4 & \multicolumn{4}{|c|}{ background for penetrating $\mathrm{He}$} & - & 5 \\
\hline 5 & \multicolumn{4}{|c|}{ background for penetrating $Z>2$} & - & 6 \\
\hline 6 & -1 & - & 0.7 & 1.4 & 1 & 1 \\
\hline 7 & -1 & - & 1.4 & 2.8 & 1 & 1 \\
\hline 8 & -1 & - & 2.8 & $\sim 6$ & 1 & 1 \\
\hline 9 & 1 & 1 & 13.3 & 15.0 & 2 & 1 \\
\hline 10 & 1 & 1 & 15.0 & 17.0 & 2 & 1 \\
\hline 11 & 1 & 1 & 17.0 & 19.0 & 2 & 1 \\
\hline 12 & 1 & 1 & 19.0 & 21.0 & 2 & 1 \\
\hline 13 & 1 & 1 & 21.0 & 24.0 & 2 & 1 \\
\hline 14 & 1 & 1 & 24.0 & 27.0 & 2 & 1 \\
\hline 15 & 1 & 1 & 27.0 & 30.0 & 2 & 1 \\
\hline 16 & 1 & 1 & 30.0 & 33.0 & 2 & 1 \\
\hline 17 & 1 & 1 & 33.0 & 36.0 & 2 & 1 \\
\hline 18 & 1 & 1 & 36.0 & 40.0 & 2 & 1 \\
\hline 19 & 2 & 3 & 17.0 & 21.0 & 3 & 2 \\
\hline 20 & 2 & 3 & 21.0 & 27.0 & 3 & 2 \\
\hline
\end{tabular}




\begin{tabular}{|c|c|c|c|c|c|c|}
\hline $\begin{array}{l}\text { SW } \\
\text { Ctr \# }\end{array}$ & $Z$ & $A$ & $\begin{array}{l}\text { KEmin } \\
(\mathrm{MeV} / \mathrm{n})\end{array}$ & $\begin{array}{l}\text { KEmax } \\
(\mathrm{MeV} / \mathrm{n})\end{array}$ & $\begin{array}{l}\text { Particle } \\
\text { type }\end{array}$ & Cat. \\
\hline 21 & -2 & 3 & 27.0 & 33.0 & 3 & 2 \\
\hline 22 & -2 & 3 & 33.0 & 40.0 & 3 & 2 \\
\hline 23 & -2 & 3 & 40.0 & 47.0 & 3 & 2 \\
\hline 24 & -2 & 4 & 13.3 & 15.0 & 4 & 2 \\
\hline 25 & -2 & 4 & 15.0 & 17.0 & 4 & 2 \\
\hline 26 & -2 & 4 & 17.0 & 19.0 & 4 & 2 \\
\hline 27 & -2 & 4 & 19.0 & 21.0 & 4 & 2 \\
\hline 28 & -2 & 4 & 21.0 & 24.0 & 4 & 2 \\
\hline 29 & -2 & 4 & 24.0 & 27.0 & 4 & 2 \\
\hline 30 & -2 & 4 & 27.0 & 30.0 & 4 & 2 \\
\hline 31 & -2 & 4 & 30.0 & 33.0 & 4 & 2 \\
\hline 32 & -2 & 4 & 33.0 & 36.0 & 4 & 2 \\
\hline 33 & -2 & 4 & 36.0 & 40.0 & 4 & 2 \\
\hline 34 & -6 & 12 & 26.4 & 30.0 & 6 & 3 \\
\hline 35 & -6 & 12 & 30.0 & 33.0 & 6 & 3 \\
\hline 36 & -6 & 12 & 33.0 & 36.0 & 6 & 3 \\
\hline 37 & -6 & 12 & 36.0 & 40.0 & 6 & 3 \\
\hline 38 & -6 & 12 & 40.0 & 45.0 & 6 & 3 \\
\hline 39 & -6 & 12 & 45.0 & 52.0 & 6 & 3 \\
\hline 40 & -6 & 12 & 52.0 & 62.0 & 6 & 3 \\
\hline 41 & -6 & 12 & 62.0 & 74.0 & 6 & 3 \\
\hline 42 & -8 & 16 & 30.0 & 33.0 & 8 & 3 \\
\hline 43 & 8 & 16 & 33.0 & 36.0 & 8 & 3 \\
\hline 44 & 8 & 16 & 36.0 & 40.0 & 8 & 3 \\
\hline 45 & 8 & 16 & 40.0 & 45.0 & 8 & 3 \\
\hline 46 & 8 & 16 & 45.0 & 52.0 & 8 & 3 \\
\hline 47 & 8 & 16 & 52.0 & 62.0 & 8 & 3 \\
\hline 48 & 8 & 16 & 62.0 & 74.0 & 8 & 3 \\
\hline 49 & 8 & 16 & 74.0 & 87.0 & 8 & 3 \\
\hline 50 & 10 & 20 & 33.0 & 36.0 & 10 & 3 \\
\hline 51 & 10 & 20 & 36.0 & 40.0 & 10 & 3 \\
\hline 52 & 10 & 20 & 40.0 & 45.0 & 10 & 3 \\
\hline 53 & 10 & 20 & 45.0 & 52.0 & 10 & 3 \\
\hline 54 & 10 & 20 & 52.0 & 62.0 & 10 & 3 \\
\hline 55 & 10 & 20 & 62.0 & 74.0 & 10 & 3 \\
\hline 56 & 10 & 20 & 74.0 & 87.0 & 10 & 3 \\
\hline 57 & 10 & 20 & 87.0 & 98.0 & 10 & 3 \\
\hline 58 & 12 & 24 & 40.0 & 45.0 & 12 & 3 \\
\hline 59 & 12 & 24 & 45.0 & 52.0 & 12 & 3 \\
\hline 60 & 12 & 24 & 52.0 & 62.0 & 12 & 3 \\
\hline 61 & 12 & 24 & 62.0 & 74.0 & 12 & 3 \\
\hline 62 & 12 & 24 & 74.0 & 87.0 & 12 & 3 \\
\hline 63 & 12 & 24 & 87.0 & 98.0 & 12 & 3 \\
\hline
\end{tabular}




\begin{tabular}{|c|c|c|c|c|c|c|}
\hline $\begin{array}{l}\mathrm{SW} \\
\mathrm{Ctr} \#\end{array}$ & $\mathrm{Z}$ & A & $\begin{array}{l}\text { Kemin } \\
(\mathrm{MeV} / \mathrm{n})\end{array}$ & $\begin{array}{l}\text { KEmax } \\
(\mathrm{MeV} / \mathrm{n})\end{array}$ & $\begin{array}{l}\text { Particle } \\
\text { type }\end{array}$ & Cat. \\
\hline 64 & 12 & 24 & 98.0 & 109.0 & 12 & 3 \\
\hline 65 & 14 & 28 & 40.0 & 45.0 & 14 & 3 \\
\hline 66 & 14 & 28 & 45.0 & 52.0 & 14 & 3 \\
\hline 67 & 14 & 28 & 52.0 & 62.0 & 14 & 3 \\
\hline 68 & 14 & 28 & 62.0 & 74.0 & 14 & 3 \\
\hline 69 & 14 & 28 & 74.0 & 87.0 & 14 & 3 \\
\hline 70 & 14 & 28 & 87.0 & 98.0 & 14 & 3 \\
\hline 71 & 14 & 28 & 98.0 & 109.0 & 14 & 3 \\
\hline 72 & 14 & 28 & 109.0 & 119.0 & 14 & 3 \\
\hline 73 & 26 & 56 & 52.0 & 62.0 & 26 & 3 \\
\hline 74 & 26 & 56 & 62.0 & 74.0 & 26 & 3 \\
\hline 75 & 26 & 56 & 74.0 & 87.0 & 26 & 3 \\
\hline 76 & 26 & 56 & 87.0 & 98.0 & 26 & 3 \\
\hline 77 & 26 & 56 & 98.0 & 109.0 & 26 & 3 \\
\hline 78 & 26 & 56 & 109.0 & 119.0 & 26 & 3 \\
\hline 79 & 26 & 56 & 119.0 & 140.0 & 26 & 3 \\
\hline 80 & 26 & 56 & 140.0 & 163.0 & 26 & 3 \\
\hline \multicolumn{7}{|c|}{; penetrating particles: } \\
\hline 81 & 1 & 1 & 40.0 & 60.0 & 2 & 4 \\
\hline 82 & 1 & 1 & 60.0 & 100.0 & 2 & 4 \\
\hline 83 & 1 & 1 & 100.0 & 200.0 & 2 & 4 \\
\hline 84 & 1 & 1 & 200.0 & 400.0 & 2 & 4 \\
\hline 85 & 1 & 1 & 400.0 & 2000.0 & 2 & 4 \\
\hline 86 & 2 & 4 & 40.0 & 60.0 & 4 & 5 \\
\hline 87 & 2 & 4 & 60.0 & 100.0 & 4 & 5 \\
\hline 88 & 2 & 4 & 100.0 & 200.0 & 4 & 5 \\
\hline \multicolumn{7}{|c|}{; H1-stopping: } \\
\hline 89 & 1 & 1 & 4.1 & 6.0 & 2 & 0 \\
\hline 90 & 1 & 1 & 6.0 & 8.0 & 2 & 0 \\
\hline 91 & 1 & 1 & 8.0 & 10.0 & 2 & 0 \\
\hline 92 & 1 & 1 & 10.0 & 12.0 & 2 & 0 \\
\hline 93 & 1 & 1 & 12.0 & 14.0 & 2 & 0 \\
\hline 94 & 1 & 1 & 14.0 & 16.0 & 2 & 0 \\
\hline 95 & 2 & 4 & 5.5 & 7.0 & 4 & 0 \\
\hline 96 & 2 & 4 & 7.0 & 8.0 & 4 & 0 \\
\hline 97 & 2 & 4 & 8.0 & 9.0 & 0 & 0 \\
\hline 98 & 2 & 4 & 9.0 & 10.0 & 4 & 0 \\
\hline 99 & 2 & 4 & 10.0 & 11.0 & 4 & 0 \\
\hline 100 & 2 & 4 & 11.0 & 12.0 & 4 & 0 \\
\hline 101 & 2 & 4 & 12.0 & 13.0 & 4 & 0 \\
\hline 102 & - & - & $>13.0$ & & - & 0 \\
\hline
\end{tabular}




\begin{tabular}{|c|c|c|c|c|c|c|}
\hline $\begin{array}{l}\text { SW } \\
\text { Ctr \# }\end{array}$ & $\mathrm{Z}$ & A & $\begin{array}{l}\text { KEmin } \\
(\mathrm{MeV} / \mathrm{n})\end{array}$ & $\begin{array}{l}\text { KEmax } \\
(\mathrm{MeV} / \mathrm{n})\end{array}$ & $\begin{array}{l}\text { Particle } \\
\text { type }\end{array}$ & Cat. \\
\hline \multicolumn{7}{|c|}{; livetime counters } \\
\hline \multicolumn{7}{|c|}{103 "stopping" stim events in category 1 (stopping e $+\mathrm{H}$ ) } \\
\hline \multicolumn{7}{|c|}{104 “stopping” stim events in category 2 (stopping He) } \\
\hline \multicolumn{7}{|c|}{105 “stopping" stim events in category 3 (stopping $Z>2$ ) } \\
\hline \multicolumn{7}{|c|}{106 "penetrating" stim events in category 4 (penetrating H) } \\
\hline \multicolumn{7}{|c|}{107 "penetrating" stim events in category 5 (penetrating $\mathrm{He}$ ) } \\
\hline \multicolumn{7}{|c|}{108 "H1Only" stim events in category 0 for H1only livetime } \\
\hline
\end{tabular}

\section{References}

H.H. Andersen, J.F. Ziegler, Hydrogen Stopping Powers and Ranges in All Elements (Pergamon, New York, 1977)

R. Baker, CPU24 Microprocessor User's Manual, GSFC internal document, 2003

D.A. Bryant, T.L. Cline, U.D. Desai, F.B. McDonald, J. Geophys. Res. 67, 4983 (1962)

CCSDS Packet Telemetry Recommendation for Space Data System Standards, 102.0-B-5, Blue Book, Issue 5 (CCSDS, Washington, DC, 2000)

C.M.S. Cohen, in Solar Eruptions and Energetic Particles, ed. by N. Gopalswamy, R.A. Mewaldt, J. Torsti. AGU Geophysical Monograph, vol. 165 (2006), p. 275

C.M.S. Cohen et al., J. Geophys. Res. (2005). doi:10.1029/2005JA011004

W.R. Cook, STEREO PHASIC User's Manual, Caltech internal document, 2002

J. Luhmann et al., Adv. Space Res. 36, 1534 (2005)

J. Luhmann et al., Space Sci. Rev. (2007, this issue). doi:10.1007/s11214-007-9170-X

G.M. Mason et al., Space Sci. Rev. (2007, this issue). doi:10.1007/s-11214-006-9087-9

R.A. Mewaldt et al., 30th Int. Cosmic Ray Conf., Mérida, México (2007a)

R.A. Mewaldt et al., Space Sci. Rev. (2007b, this issue). doi:10.1007/s-11214-007-9288-X

R.A. Mewaldt et al., J. Geophys. Res. (2005). doi:10.1029/2005JA011038

R. Müller-Mellin et al., Space Sci. Rev. (2007, this issue). doi:10.1007/s11214-007-9204-4

D.V. Reames, Space Sci. Rev. 90, 413 (1999)

D.V. Reames, iIn Space Weather, ed. by P. Song, H.J. Singer, G. Siscoe. AGU Geophysical Monograph, vol. 125 (2001), p. 101

D.V. Reames, C.K. Ng, Astrophys. J. 504, 1002 (1998)

D.V. Reames, S.W. Kahler, C.K. Ng, Astrophys. J. 491, 414 (1997)

STEREO Mission Operations Center (MOC) to Payload Operations Center (POC) and to STEREO Science Center (SSC) Interface Control Document (ICD), Applied Physics Laboratory internal document 73819045A (2002)

E.C. Stone et al., Space Sci. Rev. 86, 357 (1998)

R. Tousey, in Space Research XIII, ed. by M.J. Rycroft, S.K. Runcorn (Akademie-Verlag, Berlin, 1973), p. 713

A.J. Tylka, M.A. Lee, Astrophys. J. 646, 1319 (2006)

T.T. von Rosenvinge, H.V. Cane, in Solar Eruptions and Energetic Particles, ed. by N. Gopalswamy, R.A. Mewaldt, J. Torsti. AGU Geophysical Monograph, vol. 165 (2006), p. 103 